\title{
Recurrence of intestinal metaplasia and early neoplasia after endoscopic eradication therapy for Barrett's esophagus: a systematic review and meta-analysis*
}

\section{(ㄷ)(ㄱ) $\Theta$}

\author{
Authors \\ Edmundowicz ${ }^{2}$, Dayna S. Early ${ }^{1}$, Sachin Wani ${ }^{2}$ \\ Institutions \\ 1 Washington University School of Medicine, St. Louis, \\ MO, USA \\ 2 University of Colorado Anschutz Medical Campus, \\ Aurora, CO, USA \\ 3 University of North Carolina, Chapel Hill, NC, USA \\ 4 Feinberg School of Medicine Northwestern University, \\ Chicago, IL, USA \\ 5 University of California, Los Angeles, Los Angeles, CA, \\ USA \\ 6 Arizona Center for Digestive Health, Gilbert, AZ, USA
}

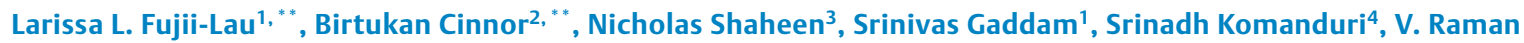
Muthusamy ${ }^{5}$, Ananya Das ${ }^{6}$, Robert Wilson ${ }^{2}$, Violette C. Simon ${ }^{2}$, Vladimir Kushnir ${ }^{1}$, Daniel Mullady ${ }^{1}$, Steven A.

submitted 22.11.2016

accepted after revision 15.2.2017

\author{
Bibliography \\ DOI https://doi.org/10.1055/s-0043-106578 | \\ Endoscopy International Open 2017; 05: E430-E449 \\ (c) Georg Thieme Verlag KG Stuttgart · New York \\ ISSN 2364-3722
}

Corresponding author

Sachin Wani, MD, Division of Gastroenterology and

Hepatology, University of Colorado Anschutz Medical

Center, Mail Stop F735, 1635 Aurora Court, Rm 2.031,

Aurora, CO 80045, USA

Fax: +1-720-848-2749

sachinwani10@yahoo.com

\section{ABSTRACT}

Background Conflicting data exist with regard to recurrence rates of intestinal metaplasia (IM) and dysplasia after achieving complete eradication of intestinal metaplasia (CE-IM) in Barrett's esophagus (BE) patients.

Aim (i) To determine the incidence of recurrent IM and dysplasia achieving CE-IM and (ii) to compare recurrence rates between treatment modalities [radiofrequency ablation (RFA) with or without endoscopic mucosal resection (EMR) vs stepwise complete EMR (SRER)].

Methods A systematic search was performed for studies reporting on outcomes and estimates of recurrence rates after achieving CE-IM. Pooled incidence [per 100-patientyears (PY)] and risk ratios with $95 \% \mathrm{Cl}$ were obtained. Heterogeneity was measured using the $R^{2}$ statistic. Subgroup analyses, decided a priori, were performed to explore heterogeneity in results.

Results A total of 39 studies were identified (25-RFA, 13SRER, and 2 combined). The pooled incidence of any recurrence was $7.5(95 \% \mathrm{Cl} 6.1-9.0) / 100 \mathrm{PY}$ with a pooled incidence of IM recurrence rate of $4.8(95 \% \mathrm{Cl} 3.8-5.9) / 100$ PY, and dysplasia recurrence rate of 2.0 ( $95 \% \mathrm{Cl} 1.5-2.5) /$ 100 PY. Compared to the SRER group, the RFA group had significantly higher overall [8.6 (6.7-10.5)/100 PY vs. 5.1 (3.1-7)/100 PY, $P=0.01]$ and IM recurrence rates [5.8 $(4.3-7.3) / 100$ PY vs. $3.1(1.7-4) / 100 \mathrm{PY}, P<0.01]$ with no difference in recurrence rates of dysplasia. Significant heterogeneity between studies was identified. The majority of recurrences were amenable to repeat endoscopic eradication therapy (EET).

Conclusion The results of this study demonstrate that the incidence rates of overall, IM, and dysplasia recurrence rates post-EET are not inconsiderable and reinforce the importance of close surveillance after achieving CE-IM.

\footnotetext{
* Results of this study were presented in part at Digestive Disease Week 2015, Washington, DC, USA

** Drs Fujii-Lau and Cinnor contributed equally to this manuscript and are joint first authors.
}

\section{Introduction}

Barrett's esophagus (BE) is the only identifiable premalignant condition for esophageal adenocarcinoma (EAC), a malignancy associated with a dismal 5 -year survival rate of $<15 \%$ and in- 
creasing annual incidence [1-7]. BE is believed to affect $1-2 \%$ of the general population and is characterized by replacement of normal squamous epithelium of the distal esophagus by a columnar lined esophagus [8-10]. Malignant transformation of $B E$ to $E A C$ is thought to occur in a stepwise and probabilistic fashion through the histopathologic stages of low grade dysplasia (LGD), then high grade dysplasia (HGD) which in turn gives rise to intramucosal carcinoma and eventually progresses to invasive adenocarcinoma [11-15].

This pathway of BE to invasive EAC provides an opportunity to halt the progression and decrease the incidence and prevalence of Barrett's related EAC and ultimately impact the morbidity and mortality related to this lethal cancer. To this end, various endoscopic eradication therapies (EET) have been evaluated over the years. Advances in this field have resulted in a significant decline in patients referred for esophagectomy with comparable outcomes (EAC free survival) reported in BE patients with HGD and mucosal EAC treated with esophagectomy and EET [16-19]. Two of such EETs that are widely used alone or in combination are endoscopic mucosal resection (EMR) and radiofrequency ablation (RFA). Efficacy data from two randomized controlled trials have shown that RFA decreases the risk of neoplastic progression among patients with BE-associated HGD and LGD $[20,21]$. Recently, data from the US multicenter RFA Patient Registry that included BE patients treated with RFA showed that $0.2 \%$ (0.7/1000 person-years) died from EAC [22]. Current contemporary management of BE-related neoplasia involves EMR of any visible lesion (if present) followed by ablation of the remaining Barrett's segment. The current goal of EET is not only to achieve complete eradication of dysplasia/ neoplasia (CE-D; including intramucosal EAC, HGD and LGD) but also complete eradication of intestinal metaplasia (CE-IM) given the $30 \%$ risk of metachronous neoplasia $[23,24]$.

The effectiveness of both techniques (EMR and RFA) in achieving CE-IM and CE-D has been demonstrated in multiple studies. Reported CE-D and CE-IM rates for EMR are in the ranges $82-100 \%$ and $72-97 \%$, respectively [25-29], and those for RFA with and without EMR are in the ranges $83-93 \%$ and $78-93 \%$, respectively [30-33]. In addition, available data indicate that most patients maintain the status of CE-IM [30, 32,33]. The high efficacy rate of EET in eradicating BE-related neoplasia and maintaining remission has revolutionized the management of these patients avoiding the morbidity and mortality associated with esophagectomy. However, the focus has now shifted to the durability of EET. Patients with BE-related neoplasia undergoing EET and achieving CE-IM are at persistent risk for recurrent IM and dysplasia; however, precise estimates of recurrence rates are not available. Widely variable recurrence rates have been reported after CE-IM in patients undergoing EMR alone [26-29], as well as RFA with or without EMR (dysplasia: $0-15 \%$, IM: $7-39.5 \%$ ) [30, 33-36].

The absence of a reliable estimate of recurrence rates has made it difficult to inform patients as to the expected outcomes with EET. Additionally, guidelines with regard to surveillance and the interval of surveillance for patients who have achieved CE-IM following EET currently rely on expert opinion. To this end, the aim of this systematic review and meta-analysis was to determine the incidence of recurrence of IM and dysplasia post CE-IM for patients with BE treated with EET (EMR, RFA or a combination of both).

\section{Materials and methods}

This systematic review and meta-analysis was performed and reported according to the recommendations of the Preferred Reporting Items for Systematic Reviews and Meta-Analyses (PRISMA) criteria [37].

\section{Search strategy}

Three databases (MEDLINE PubMed, EMBASE, and Web of Science) were systematically searched (by author LLFL) for relevant articles from 1996 through 31 May 2016. Keywords used in the search included a combination of "Barrett's esophagus, Barrett's oesophagus, recurrence, response, eradication, outcomes, complete, endoscopic ablation, radiofrequency ablation, endoscopic resection, endoscopic mucosal resection, subsquamous Barrett's, subsquamous intestinal metaplasia." Reference lists of pertinent articles, including the selected studies for inclusion, reviews, and practice guidelines, were also manually searched to identify any other potentially relevant articles. Relevant abstracts identified by EMBASE from the major gastroenterology conferences including Digestive Disease Week, American College of Gastroenterology, and United European Gastroenterology published within the past 5 years were also included.

\section{Study inclusion and exclusion criteria and definitions}

Each abstract identified by the search strategy was reviewed by two independent authors (LLFL and BC) for possible inclusion in the study. Full text manuscripts were obtained and evaluated for those that were potentially relevant after abstract review. Studies were included if they met the following strict criteria: (1) study design - randomized control trial, cohort studies, case series with at least 20 patients; (2) patient population patients who achieved CE-IM after endoscopic therapy of Barrett's esophagus with IM, dysplasia or early adenocarcinoma (EAC); (3) intervention - primary endoscopic therapy with stepwise complete endoscopic resection (SRER) or radiofrequency ablation (RFA) with or without focal endoscopic mucosal resection (EMR); (4) outcome - reported number of patients with recurrent IM, dysplasia, or EAC on histology; and (5) mean followup of at least 1 year after the first endoscopy confirming complete eradication. The definition of $\mathrm{CE}$ required both endoscopic and pathologic absence of IM and dysplasia during at least one endoscopic evaluation after EET. Recurrence was defined by the presence of IM and/or dysplasia in the esophagus and/or gastroesophageal junction/cardia after achieving CEIM. IM obtained on biopsies of the gastric cardia alone (separate from gastroesophageal junction biopsies) was not considered a recurrence.

Studies were excluded from the final analysis if any of the following was present: (1) study design of case-control, case series with less than 20 patients, case reports, cross-sectional 
studies, editorials, letters to the editor, reviews, book chapters; (2) a patient population which did not have pathologic confirmation (of the initial histologic reading before EET and recurrence) by a second or specialized pathologist, the manuscript did not specify how many patients failed endoscopic therapy (did not achieve CE-IM) or required surgical treatment; (3) other forms of primary endoscopic therapy including cryotherapy, photodynamic therapy, argon plasma coagulation; (4) studies with insufficient data to estimate an incidence of recurrence; (5) non-English reports; (6) non-human or non-clinical studies; and (7) duplicate reports. In the event where multiple manuscripts included the same cohort of patients, the study with the longest mean follow-up interval was included.

\section{Data abstraction}

Data extraction using standardized forms was independently performed by two investigators (LLFL and BC). Any disagreement over the extracted data was resolved by consensus (LLFL, $B C$ and SW). The following data were collected from each study: (1) study characteristics - design (cohort, randomized control trial, case series), outlook (retrospective, prospective), setting (single center or multicenter), tertiary centers vs community, location (USA vs non-USA); (2) patient characteristics - demographics (mean age, proportion of males, average maximal BE length, highest pretreatment histological grade), total number of patients who underwent EET, proportion of patients with follow-up for at least 1 year, if and how patients who were lost to follow-up were accounted for; (3) treatment characteristicsprimary endoscopic treatment with SRER or RFA with or without focal EMR, definition of CE; (4) surveillance methodologywhether the neo-gastroesophageal junction/gastric cardia was biopsied separately, biopsy methods (1- or 2-cm intervals; if the study allowed biopsies every $1-2 \mathrm{~cm}$, it was included in the 2-cm cohort); and (5) outcomes data including mean or median length of follow-up for the study (years) and number of events of recurrent IM, dysplasia and EAC. If a study included patients who had more than one surveillance upper endoscopy with recurrent IM or dysplasia, only the first endoscopy demonstrating recurrence was included.

\section{Study quality assessment}

Quality assessment of each study was independently reviewed by the same two investigators using the Downs and Black quality assessment checklist [38]. This instrument was chosen as it has been validated and assesses both randomized and non-randomized (observational) studies. If recurrence after EET was a secondary objective of the manuscript and was only reported as a descriptive analysis, then the study was designated of low quality to answer the aim of this meta-analysis. As recurrence was not considered to be a main outcome in these studies, a " 0 " was given to the following criteria that focused on the main finding: (1) are the distributions of principal confounders in each group of subjects to be compared clearly described, (2) does the study provide estimates of the random variability in the data for the main outcome, (3) have actual probability values been reported for the main outcome, (4) were the statistical tests used to assess the main outcomes appropriate, (5) were the main outcome measures used accurate, (6) was there adequate adjustment for confounding in the analysis from which the main findings were drawn, and (7) did the study have sufficient power to detect a clinically important effect where the probability value for a difference being due to change is $<5 \%$. Scores $<15,15-19$, and $>20$ were considered to be low, moderate, and high quality studies, respectively. All abstracts and case series were considered to be of low quality.

\section{Outcomes assessed}

The primary analysis focused on the pooled incidence [per 100 patient years (PY)] of IM and early neoplasia (a combination of both dysplasia and EAC for this analysis) in patients achieving CE-IM after EET. Subgroup analyses were performed for hypotheses determined a priori that may explain potential heterogeneity between the included studies. The subgroup analyses designated a priori were type of report (abstract vs full text), study outlook (prospective vs retrospective), study setting (single center vs multicenter), hospital type (solely tertiary centers vs mixed), study location (USA vs non-USA), quality (divided into high, medium and low), type of EET (SRER vs RFA with or without EMR), whether non-dysplastic BE patients were included in the treatment group, CE definition (classified as 1 vs 2 negative endoscopies), whether the neo-squamous gastroesophageal junction/gastric cardia was biopsied separately during surveillance examinations, length intervals of surveillance biopsies ( 1 vs $2 \mathrm{~cm}$; studies that allowed biopsies every $1-2 \mathrm{~cm}$ were classified in the latter group), and length of Barrett's segment $(\leq 3 \mathrm{~cm}$ vs $>3 \mathrm{~cm}$ ). A cutoff of $3 \mathrm{~cm}$ was used for BE length as most studies used this to differentiate between short and long segment BE. Due to the variability in reporting on continuous variables such as BE length, changing the variable to a binary variable was deemed to be the most appropriate method to include the maximum number of studies in this analysis.

\section{Statistical analysis}

All analyses and graphs were performed using Comprehensive Meta-Analysis Version 2 or 3 (Biostat, Englewood, NJ, United States). The random effects model, as described by DerSimonian and Laird, was used for all analyses to determine incidence of recurrence (number of events per $100 \mathrm{PY}$ ) with the $95 \%$ confidence intervals $(\mathrm{Cl})$ [39]. Heterogeneity was assessed using the $I^{2}$ statistic, with a value $>50$ considered significant for heterogeneity and was the reason we used the random effects model. Sensitivity analysis was performed to determine whether a single study had significant influence on the results, focusing on the total recurrences for both IM and dysplasia. Publication bias was assessed using a funnel plot with event rate plotted against standard error Egger's regression intercept ( $P$ values $\leq 0.10$ suggestive of significant publication bias). Descriptive analyses were used for the risk of recurrence given the small number of studies reporting this information and the heterogeneity of the risk factors included. 


\section{Results}

\section{Characteristics of included studies}

From the original search, of the 3311 identified studies, 3167 were excluded based on the title and abstract alone ( $\vee$ Fig. 1). The full-text of the remaining 144 studies was reviewed and an additional 111 studies were excluded. We updated our search for eligible studies after the 2016 Digestive Disease Week conference and identified three more abstracts that met our study's inclusion and exclusion criteria. A total of 39 studies (33 full-text manuscripts and 6 abstracts) were included in the final analysis. The two groups in a randomized controlled trial comparing SRER to RFA with focal EMR were treated as separate patient cohorts in this analysis [34].

The overall study and patient characteristics are highlighted in > Table 1 and $>$ Table 2, respectively. A total of 25 studies focused on RFA with or without EMR, 13 studies described outcomes in patients treated with SRER as the primary EET modality ( 1 study compared both RFA and SRER), and 2 abstracts combined both SRER and RFA in their analysis. Of the manuscripts focusing on RFA, 1 was a randomized controlled trial, 19 cohort observational studies, and 5 case series. Two cohort studies were included in only the recurrent dysplasia analysis as they did not report how many patients had recurrent IM [40, 41]. The distribution of studies based on geographic location was as follows: USA (23), Europe (13), Australia (2), and Canada (1). Overall, 4355 patients (3213 treated with RFA, 567 with SRER, and 575 both modalities) were included in the analysis for a total of 11837.63 patient-years of follow-up.

\section{Quality of the included studies}

In all of the included randomized controlled trials and cohort studies, the main objective, intervention of interest, and main findings were clearly described. Of the 18 full text randomized controlled trials or cohort studies, 4 were considered to be of high quality (3 RFA, 1 SRER), 7 moderate quality (6 RFA studies, and 1 study comparing RFA to SRER), and 7 low quality (7 RFA) as highlighted in - Supplemental Table 1. The majority of the moderate and low quality studies reported recurrence after EET as a secondary analysis; only 7 cohort full text studies (6 RFA and 1 SRER) had recurrence as the primary outcome [27,32, $35,36,42-44]$. The 16 case series and 5 cohort abstracts were all considered to be low quality.

\section{Incidence of recurrence}

Overall, a total of 1000 recurrences occurred after CE-IM, of which 722 occurred after RFA, 103 after SRER therapy, and 175 after either therapy. Overall, the pooled incidence of any recurrence was $7.5(95 \% \mathrm{Cl} 6.1-9) / 100 \mathrm{PY}$ ( $\mathbf{F i g . 2 a})$, recurrence of IM was $4.8(3.8-5.9) / 100$ PY ( $\triangleright$ Fig. $2 b)$, and recurrence of dysplasia was $2.0(1.5-2.5) / 100 \mathrm{PY}(\triangleright$ Fig. $2 \mathbf{c}$ ). The total recurrence does not equal the combination of both IM and dysplasia recurrence as some studies only reported on either IM or dysplasia recurrence and these were not included in the total recurrences.
3111 studies identified during systematic search

3167 excluded:

1173- lack of relevance (not BE)

1087- review/meta-analysis

300 - lack of relevance (BE studies)

290- case report, letters, editorial

148- basic science

96- other primary endoscopic therapy

41- irrelevant abstract

20- study of other visualization techniques

15- duplicate

\section{4 full-text articles reviewed}

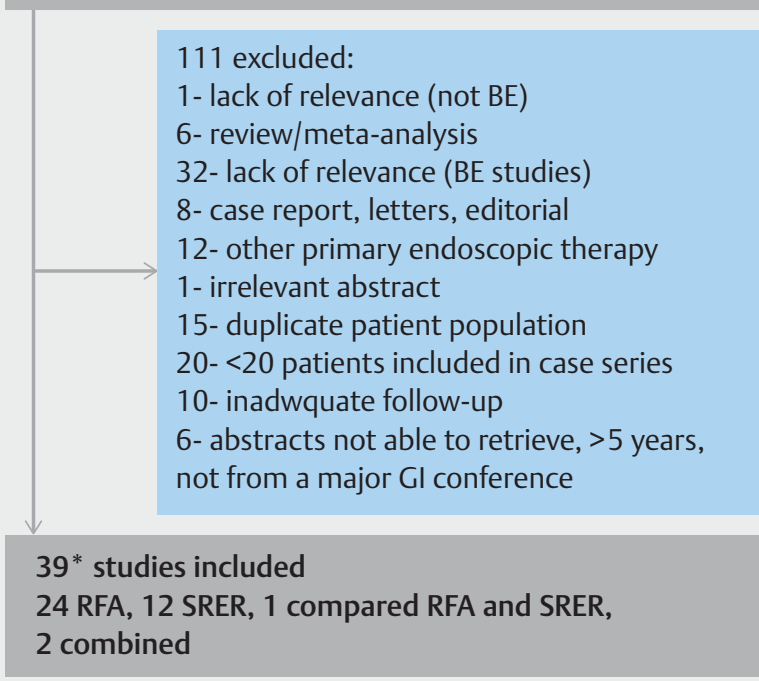

- Fig. 1 Study flow diagram. *133 full text studies and 6 abstracts.

The IM and dysplasia recurrence rates for the RFA and SRER groups are highlighted in > Table 3 . Compared to the SRER group, the RFA group had significantly higher overall [8.6 $(6.7-10.5) / 100$ PY vs $4.9(3-6.8) / 100$ PY, $P=0.007]$ and IM recurrence rates $[5.8(4.3-7.3) / 100$ PY vs $3.3(2-4.7) / 100$ PY, $P$ $=0.015]$. However, there was no difference in the recurrence rate of dysplasia between the two groups $[1.9(1.3-2.5) / 100$ $P Y$ in the RFA vs $1.2(0.4-1.9) / 100$ PY in the SRER group, $P=$ $0.11]$. The majority of recurrences were amenable to repeat EET as highlighted in > Table 4.

\section{Subgroup analyses on incidence of recurrence}

Significant heterogeneity between studies was identified, with an overall $R^{2}$ value of 86 . To explore the potential causes of this heterogeneity, several subgroup analyses were performed. In the RFA group, significant factors associated with a higher IM recurrence rate included retrospective study design, inclusion of abstracts, surveillance biopsy protocol and BE length of $\leq 3 \mathrm{~cm}$ ( $\triangleright$ Table 5 ). Similar subgroup analyses performed in the SRER group are highlighted in $\bullet$ Table 6. 
- Table 1 Study characteristics.

\begin{tabular}{|c|c|c|c|c|c|c|c|c|c|c|c|}
\hline Study & $\begin{array}{l}\text { EET } \\
\text { type }\end{array}$ & $\begin{array}{l}\text { Manu- } \\
\text { script } \\
\text { type }\end{array}$ & $\begin{array}{l}\text { De- } \\
\text { sign }\end{array}$ & $\begin{array}{l}\text { Set- } \\
\text { ting }\end{array}$ & Outlook & $\begin{array}{l}\text { Loca- } \\
\text { tion }\end{array}$ & Quality & $\begin{array}{l}\text { Histology } \\
\text { included }\end{array}$ & $\begin{array}{l}\text { No. of } \\
\text { EGD } \\
\text { for CR }\end{array}$ & $\begin{array}{l}\text { Cardia } \\
\text { biop- } \\
\text { sied }\end{array}$ & $\begin{array}{l}\text { Biopsy } \\
\text { inter- } \\
\text { vals, cm }\end{array}$ \\
\hline $\begin{array}{l}\text { Fleischer et al., } \\
2010 \text { [52] }\end{array}$ & RFA & Full text & $\begin{array}{l}\text { Co- } \\
\text { hort }\end{array}$ & Multi & $\begin{array}{l}\text { Prospec- } \\
\text { tive }\end{array}$ & USA & $\begin{array}{l}\text { Moder- } \\
\text { ate }\end{array}$ & IM & 2 & No & 1 \\
\hline $\begin{array}{l}\text { Alvarez Herrero } \\
\text { et al., } 2011 \text { [53] }\end{array}$ & RFA & Full text & CS & Multi & $\begin{array}{l}\text { Prospec- } \\
\text { tive }\end{array}$ & Europe & Low & L,H,EAC & 1 & Yes & 2 \\
\hline $\begin{array}{l}\text { Shaheen et al., } \\
2011 \text { [30] }\end{array}$ & RFA & Full text & RCT & Multi & $\begin{array}{l}\text { Prospec- } \\
\text { tive }\end{array}$ & USA & $\begin{array}{l}\text { Moder- } \\
\text { ate }\end{array}$ & $\mathrm{L}, \mathrm{H}$ & 1 & No & 1 \\
\hline $\begin{array}{l}\text { Vaccaro et al., } \\
2011 \text { [36] }\end{array}$ & RFA & Full text & $\begin{array}{l}\text { Co- } \\
\text { hort }\end{array}$ & Single & $\begin{array}{l}\text { Retro- } \\
\text { spective }\end{array}$ & USA & $\begin{array}{l}\text { Moder- } \\
\text { ate }\end{array}$ & IM,L,H,EAC & 1 & Yes & NR \\
\hline $\begin{array}{l}\text { van Vilsteren et } \\
\text { al., } 2011 \text { [34] }\end{array}$ & Both & Full text & $\mathrm{RCT}$ & Multi & $\begin{array}{l}\text { Prospec- } \\
\text { tive }\end{array}$ & Europe & $\begin{array}{l}\text { Moder- } \\
\text { ate }\end{array}$ & $\mathrm{H}, \mathrm{EAC}$ & 1 & Yes & 2 \\
\hline $\begin{array}{l}\text { Caillol et al., } \\
2012 \text { [54] }\end{array}$ & RFA & Full text & $\begin{array}{l}\text { Co- } \\
\text { hort }\end{array}$ & Single & $\begin{array}{l}\text { Retro- } \\
\text { spective }\end{array}$ & Europe & Low & IM,L,H & NR & No & 2 \\
\hline $\begin{array}{l}\text { Gupta N } 2012 \\
\text { [55] }\end{array}$ & RFA & Abstract & $\begin{array}{l}\text { Co- } \\
\text { hort }\end{array}$ & Multi & $\begin{array}{l}\text { Retro- } \\
\text { spective }\end{array}$ & USA & Low & $\mathrm{H}, \mathrm{EAC}$ & NR & NR & NR \\
\hline $\begin{array}{l}\text { van Vilsteren et } \\
\text { al., } 2012 \text { [56] }\end{array}$ & RFA & Full text & CS & Single & $\begin{array}{l}\text { Retro- } \\
\text { spective }\end{array}$ & Europe & Low & $\mathrm{H}, \mathrm{EAC}$ & 1 & Yes & 2 \\
\hline $\begin{array}{l}\text { Akiyama et al., } \\
2013 \text { [45] }\end{array}$ & RFA & Abstract & $\begin{array}{l}\text { Co- } \\
\text { hort }\end{array}$ & Single & $\begin{array}{l}\text { Retro- } \\
\text { spective }\end{array}$ & USA & Low & IM,L,H,EAC & 1 & NR & NR \\
\hline $\begin{array}{l}\text { Dulai et al., } \\
2013 \text { [57] }\end{array}$ & RFA & Full text & $\begin{array}{l}\text { Co- } \\
\text { hort }\end{array}$ & Single & $\begin{array}{l}\text { Retro- } \\
\text { spective }\end{array}$ & USA & Low & IM,L,H,EAC & 1 & No & 1 \\
\hline $\begin{array}{l}\text { Ertanet al., } \\
2013 \text { [58] }\end{array}$ & RFA & Full text & $\begin{array}{l}\text { Co- } \\
\text { hort }\end{array}$ & Single & $\begin{array}{l}\text { Retro- } \\
\text { spective }\end{array}$ & USA & Low & $\mathrm{L}, \mathrm{H}$ & NR & No & 1 \\
\hline $\begin{array}{l}\text { Gupta et al., } \\
2013 \text { [35] }\end{array}$ & RFA & Full text & $\begin{array}{l}\text { Co- } \\
\text { hort }\end{array}$ & Multi & $\begin{array}{l}\text { Retro- } \\
\text { spective }\end{array}$ & USA & High & IM,L,H,EAC & 2 & No & $1-2$ \\
\hline $\begin{array}{l}\text { Haidry et al., } \\
2013 \text { [59] }\end{array}$ & RFA & Full text & $\begin{array}{l}\text { Co- } \\
\text { hort }\end{array}$ & Multi & $\begin{array}{l}\text { Retro- } \\
\text { spective }\end{array}$ & Europe & Low & L,H,EAC & 1 & No & NR \\
\hline $\begin{array}{l}\text { Korst et al., } \\
2013 \text { [60] }\end{array}$ & RFA & Full text & CS & Single & $\begin{array}{l}\text { Retro- } \\
\text { spective }\end{array}$ & USA & Low & IM,L,H,EAC & NR & Yes & $1-2$ \\
\hline $\begin{array}{l}\text { Orman et al., } \\
2013 \text { [42] }\end{array}$ & RFA & Full text & $\begin{array}{l}\text { Co- } \\
\text { hort }\end{array}$ & Single & $\begin{array}{l}\text { Retro- } \\
\text { spective }\end{array}$ & USA & High & L,H,EAC & 1 & Yes & 1 \\
\hline $\begin{array}{l}\text { Phoa et al., } \\
2013 \text { [33] }\end{array}$ & RFA & Full text & CS & Single & $\begin{array}{l}\text { Retro- } \\
\text { spective }\end{array}$ & Europe & Low & $\mathrm{H}$,EAC & NR & Yes & 2 \\
\hline $\begin{array}{l}\text { Shue et al., } \\
2013 \text { [47] }\end{array}$ & RFA & Abstract & $\begin{array}{l}\text { Co- } \\
\text { hort }\end{array}$ & Single & $\begin{array}{l}\text { Retro- } \\
\text { spective }\end{array}$ & USA & Low & IM,L,H,EAC & 1 & NR & 2 \\
\hline $\begin{array}{l}\text { Pasricha et al., } \\
2014 \text { [32] }\end{array}$ & RFA & Full text & $\begin{array}{l}\text { Co- } \\
\text { hort }\end{array}$ & Multi & $\begin{array}{l}\text { Retro- } \\
\text { spective }\end{array}$ & USA & $\begin{array}{l}\text { Moder- } \\
\text { ate }\end{array}$ & IM,L,H,EAC & 1 or 2 & No & 1 \\
\hline $\begin{array}{l}\text { Strauss et al., } \\
2014 \text { [61] }\end{array}$ & RFA & Full text & $\begin{array}{l}\text { Co- } \\
\text { hort }\end{array}$ & Multi & $\begin{array}{l}\text { Retro- } \\
\text { spective }\end{array}$ & USA & $\begin{array}{l}\text { Moder- } \\
\text { ate }\end{array}$ & EAC & NR & NR & NR \\
\hline $\begin{array}{l}\text { Agoston et al., } \\
2015 \text { [40] }\end{array}$ & RFA & Full text & $\begin{array}{l}\text { Co- } \\
\text { hort }\end{array}$ & Multi & $\begin{array}{l}\text { Retro- } \\
\text { spective }\end{array}$ & USA & Low & EAC & 1 & NR & 1 \\
\hline $\begin{array}{l}\text { Cotton et al., } \\
2015 \text { [43] }\end{array}$ & RFA & Full text & $\begin{array}{l}\text { Co- } \\
\text { hort }\end{array}$ & Single & $\begin{array}{l}\text { Retro- } \\
\text { spective }\end{array}$ & USA & $\begin{array}{l}\text { Moder- } \\
\text { ate }\end{array}$ & IM,L,H,EAC & 1 & Yes & 1 \\
\hline $\begin{array}{l}\text { Lada et al., } \\
2014 \text { [62] }\end{array}$ & RFA & Full text & $\begin{array}{l}\text { Co- } \\
\text { hort }\end{array}$ & Single & $\begin{array}{l}\text { Retro- } \\
\text { spective }\end{array}$ & USA & Low & $\mathrm{H}, \mathrm{EAC}$ & 2 & NR & 1 \\
\hline $\begin{array}{l}\text { Le Page et al., } \\
2016 \text { [63] }\end{array}$ & RFA & Full text & $\begin{array}{l}\text { Co- } \\
\text { hort }\end{array}$ & Single & $\begin{array}{l}\text { Retro- } \\
\text { spective }\end{array}$ & Europe & Low & $\mathrm{H}, \mathrm{EAC}$ & NR & NR & NR \\
\hline $\begin{array}{l}\text { Phoa et al., } \\
2015 \text { [64] }\end{array}$ & RFA & Full text & CS & Multi & $\begin{array}{l}\text { Prospec- } \\
\text { tive }\end{array}$ & Europe & Low & IM,L,H,EAC & 1 & Yes & 2 \\
\hline
\end{tabular}


- Table 1 (Continuation)

\begin{tabular}{|c|c|c|c|c|c|c|c|c|c|c|c|}
\hline Study & $\begin{array}{l}\text { EET } \\
\text { type }\end{array}$ & $\begin{array}{l}\text { Manu- } \\
\text { script } \\
\text { type }\end{array}$ & $\begin{array}{l}\text { De- } \\
\text { sign }\end{array}$ & $\begin{array}{l}\text { Set- } \\
\text { ting }\end{array}$ & Outlook & $\begin{array}{l}\text { Loca- } \\
\text { tion }\end{array}$ & Quality & $\begin{array}{l}\text { Histology } \\
\text { included }\end{array}$ & $\begin{array}{l}\text { No. of } \\
\text { EGD } \\
\text { for CR }\end{array}$ & $\begin{array}{l}\text { Cardia } \\
\text { biop- } \\
\text { sied }\end{array}$ & $\begin{array}{l}\text { Biopsy } \\
\text { inter- } \\
\text { vals, cm }\end{array}$ \\
\hline $\begin{array}{l}\text { Small et al., } \\
2015 \text { [44] }\end{array}$ & RFA & Full text & $\begin{array}{l}\text { Co- } \\
\text { hort }\end{array}$ & Single & $\begin{array}{l}\text { Retro- } \\
\text { spective }\end{array}$ & USA & High & $\mathrm{H}, \mathrm{EAC}$ & 1 & NR & NR \\
\hline $\begin{array}{l}\text { Giovannini } \\
\text { et al., } 2004 \text { [65] }\end{array}$ & EMR & Full text & CS & Single & $\begin{array}{l}\text { Retro- } \\
\text { spective }\end{array}$ & Europe & Low & $\mathrm{H}, \mathrm{EAC}$ & NR & No & NR \\
\hline $\begin{array}{l}\text { Larghi et al., } \\
2007 \text { [66] }\end{array}$ & EMR & Full text & CS & Multi & $\begin{array}{l}\text { Retro- } \\
\text { spective }\end{array}$ & USA & Low & $\mathrm{H}, \mathrm{EAC}$ & NR & No & $1-2$ \\
\hline $\begin{array}{l}\text { Lopes et al., } \\
2007 \text { [67] }\end{array}$ & EMR & Full text & CS & Single & $\begin{array}{l}\text { Retro- } \\
\text { spective }\end{array}$ & Europe & Low & $\mathrm{H}, \mathrm{EAC}$ & NR & No & NR \\
\hline $\begin{array}{l}\text { Chennat et al., } \\
2009 \text { [68] }\end{array}$ & EMR & Full text & CS & Single & $\begin{array}{l}\text { Retro- } \\
\text { spective }\end{array}$ & USA & Low & $\mathrm{H}, \mathrm{EAC}$ & NR & No & $1-2$ \\
\hline $\begin{array}{l}\text { Brahmania } \\
\text { et al., } 2010 \text { [69] }\end{array}$ & EMR & Full text & CS & Single & $\begin{array}{l}\text { Retro- } \\
\text { spective }\end{array}$ & Canada & Low & IM,L,H,EAC & NR & NR & NR \\
\hline $\begin{array}{l}\text { Moss et al., } \\
2010 \text { [70] }\end{array}$ & EMR & Full text & CS & Multi & $\begin{array}{l}\text { Prospec- } \\
\text { tive }\end{array}$ & $\begin{array}{l}\text { Austra- } \\
\text { lia }\end{array}$ & Low & $\mathrm{H}, \mathrm{EAC}$ & NR & No & 1 \\
\hline $\begin{array}{l}\text { Chung et al., } \\
2011 \text { [26] }\end{array}$ & EMR & Full text & CS & Multi & $\begin{array}{l}\text { Retro- } \\
\text { spective }\end{array}$ & $\begin{array}{l}\text { Austra- } \\
\text { lia }\end{array}$ & Low & $\mathrm{H}, \mathrm{EAC}$ & NR & No & 1 \\
\hline $\begin{array}{l}\text { Gerke et al., } \\
2011 \text { [25] }\end{array}$ & EMR & Full text & CS & Single & $\begin{array}{l}\text { Retro- } \\
\text { spective }\end{array}$ & USA & Low & $\mathrm{L}, \mathrm{H}, \mathrm{EAC}$ & NR & Yes & NR \\
\hline $\begin{array}{l}\text { Anders et al., } \\
2014 \text { [27] }\end{array}$ & EMR & Full text & $\begin{array}{l}\text { Co- } \\
\text { hort }\end{array}$ & Multi & $\begin{array}{l}\text { Retro- } \\
\text { spective }\end{array}$ & Europe & High & $\mathrm{L}, \mathrm{H}, \mathrm{EAC}$ & 2 & No & NR \\
\hline $\begin{array}{l}\text { Conio et al., } \\
2014 \text { [71] }\end{array}$ & EMR & Full text & CS & Single & $\begin{array}{l}\text { Retro- } \\
\text { spective }\end{array}$ & Europe & Low & $\mathrm{H}, \mathrm{EAC}$ & NR & Yes & NR \\
\hline $\begin{array}{l}\text { Konda et al., } \\
2014 \text { [28] }\end{array}$ & EMR & Full text & CS & Single & $\begin{array}{l}\text { Retro- } \\
\text { spective }\end{array}$ & USA & Low & $\mathrm{L}, \mathrm{H}, \mathrm{EAC}$ & 1 & Yes & NR \\
\hline $\begin{array}{l}\text { Belghazi et al., } \\
2016 \text { [72] }\end{array}$ & EMR & Abstract & CS & Multi & $\begin{array}{l}\text { Retro- } \\
\text { spective }\end{array}$ & Europe & Low & $\mathrm{H}, \mathrm{EAC}$ & NR & Yes & NR \\
\hline $\begin{array}{l}\text { Wani et al., } \\
2016 \text { [51] }\end{array}$ & Both & Abstract & $\begin{array}{l}\text { Co- } \\
\text { hort }\end{array}$ & Multi & $\begin{array}{l}\text { Retro- } \\
\text { spective }\end{array}$ & USA & Low & $\mathrm{L}, \mathrm{H}, \mathrm{EAC}$ & NR & NR & NR \\
\hline $\begin{array}{l}\text { Waxman et al., } \\
2016 \text { [73] }\end{array}$ & Both & Abstract & $\begin{array}{l}\text { Co- } \\
\text { hort }\end{array}$ & Multi & $\begin{array}{l}\text { Retro- } \\
\text { spective }\end{array}$ & USA & Low & L,H,EAC & NR & NR & NR \\
\hline \multicolumn{12}{|c|}{$\begin{array}{l}\text { EET = endoscopic eradication therapy, } E G D=\text { esophagogastroduodenoscopy, } C R=\text { complete remission, } E M R=\text { endoscopic mucosal resection, } R F A=\text { radiofrequency } \\
\text { ablation, } C S=\text { case series, } I M=\text { intestinal metaplasia, } L=\text { low grade dysplasia, } H=\text { high grade dysplasia, } E A C=\text { early adenocarcinoma, NR=not reported, } R C T=\text { random- } \\
\text { ized control trial. }\end{array}$} \\
\hline
\end{tabular}

\section{Sensitivity analysis}

A jackknife sensitivity analysis removing one study at a time did not change the incidence of total recurrence in the remaining studies within the RFA or SRER groups, indicating that no one study had a significant impact on the results (data not shown).

\section{Risk factors for recurrence}

Risk factors for recurrence after RFA therapy were reported in 7 studies (6 manuscripts, 1 abstract) [32, 35, 40, 42, 44-46]. A single study reported that the presence of erosive esophagitis increased the risk of recurrence on multivariable regression analysis controlling for hiatal hernia length and presence of dysplasia [hazard ratio $15.41(95 \% \mathrm{Cl} 1.65-144.33)$ ] [45]. Most studies found that age, gender, race, body mass index, baseline histology before EET, BE length, NSAID use, hiatal hernia size, and presence of a fundoplication were not associated with an increased risk of recurrence. This is in contrast to a study by Pasricha et al. that reported older age [odds ratio (OR) 1.02 per year, $95 \% \mathrm{Cl} 1.01-1.03$ ], longer $\mathrm{BE}$ segment length (OR 1.10 per $\mathrm{cm}, 95 \% \mathrm{Cl} 1.06-1.15$ ), and non-Caucasian race (OR 2.0, $95 \% \mathrm{Cl} 1.2-3.34$ ) increased the risk of recurrence on multivariable regression analysis [32]. In addition, Lada et al. found that recurrent dysplasia was more common in older patients, those with longer follow-up, and those who underwent more total procedures (including initial therapeutic interventions and surveillance endoscopies) [46]. Similarly, Small et al. found that the number of treatment sessions needed to achieve CE-IM in 
- Table 2 Patient characteristics.

\begin{tabular}{|c|c|c|c|c|c|c|c|c|c|c|}
\hline Study & $\begin{array}{l}\text { Total } \\
\text { no. }\end{array}$ & $\begin{array}{l}\text { Mean } \\
\text { age, } \\
\text { years }\end{array}$ & $\begin{array}{l}\% \\
\text { Male }\end{array}$ & $\begin{array}{l}\text { Mean } \\
\text { BE } \\
\text { length, } \\
\mathrm{cm}\end{array}$ & $\begin{array}{l}\text { Total no. } \\
\text { of pts } \\
\text { followed }\end{array}$ & $\begin{array}{l}\text { Mean } \\
\text { follow- } \\
\text { up } \\
\text { length, } \\
\text { years }\end{array}$ & $\begin{array}{l}\text { Total } \\
\text { recurren- } \\
\text { ces }\end{array}$ & $\begin{array}{l}\text { IM recur- } \\
\text { rences }\end{array}$ & $\begin{array}{l}\text { Dysplasia } \\
\text { recurren- } \\
\text { ces }\end{array}$ & $\begin{array}{l}\text { EAC recur- } \\
\text { rences }\end{array}$ \\
\hline
\end{tabular}

\section{RFA group}

\begin{tabular}{|c|c|c|c|c|c|c|c|c|c|c|}
\hline $\begin{array}{l}\text { Fleischer et al., } \\
2010 \text { [52] }\end{array}$ & 50 & 54.3 & 74 & 3.1 & 50 & 5 & 4 & 4 & 0 & 0 \\
\hline $\begin{array}{l}\text { Alvarez Herrero } \\
\text { et al., } 2011 \text { [53] }\end{array}$ & 26 & 66 & 80.8 & 11 & 20 & 2.4 & 8 & 8 & 0 & 0 \\
\hline $\begin{array}{l}\text { Shaheen et al., } \\
2011 \text { [30] }\end{array}$ & 119 & 66 & 85.7 & 4.9 & 119 & 3.05 & 19 & 14 & 3 & 2 \\
\hline $\begin{array}{l}\text { Vaccaro et al., } \\
2011 \text { [36] }\end{array}$ & 47 & 64.2 & 76.6 & 2 & 47 & 1.11 & 15 & 11 & 4 & 0 \\
\hline $\begin{array}{l}\text { van Vilsteren et } \\
\text { al., } 2011[34]^{1}\end{array}$ & 22 & 69 & 86.4 & 4 & 21 & 1.8 & 2 & 2 & 0 & 0 \\
\hline $\begin{array}{l}\text { Caillol et al., } \\
2012 \text { [54] }\end{array}$ & 34 & 60 & 82.4 & 6.8 & 34 & 1 & 2 & 0 & 0 & 2 \\
\hline $\begin{array}{l}\text { Gupta et al., } \\
2012 \text { [55] }\end{array}$ & 128 & 65.7 & 88.3 & 4.4 & 128 & 1.3 & 34 & 18 & 16 & 0 \\
\hline $\begin{array}{l}\text { van Vilsteren et } \\
\text { al., } 2012 \text { [56] }\end{array}$ & 24 & 68 & 83.3 & 8 & 20 & 1.2 & 0 & 0 & 0 & 0 \\
\hline $\begin{array}{l}\text { Akiyama et al., } \\
2013 \text { [45] }\end{array}$ & 40 & 62.5 & 80 & 4.1 & 40 & 2.18 & 7 & 7 & 0 & 0 \\
\hline $\begin{array}{l}\text { Dulai et al., } \\
2013 \text { [57] }\end{array}$ & 72 & 66.9 & 80.6 & 7.6 & 57 & 3.25 & 11 & 11 & 0 & 0 \\
\hline $\begin{array}{l}\text { Ertan et al., } \\
2013 \text { [58] }\end{array}$ & 53 & 65.4 & 73.6 & 5.7 & 47 & 2.75 & 3 & 3 & 0 & 0 \\
\hline $\begin{array}{l}\text { Gupta et al., } \\
2013 \text { [35] }\end{array}$ & 448 & 64 & 85 & 4.3 & 192 & 1.12 & 37 & 29 & 8 & 0 \\
\hline $\begin{array}{l}\text { Haidry et al., } \\
2013 \text { [59] }\end{array}$ & 335 & 68.1 & 80.9 & 5.8 & 256 & 1.58 & 37 & 17 & 16 & 4 \\
\hline $\begin{array}{l}\text { Korst et al., } \\
2013[60]\end{array}$ & 53 & 59 & 69.8 & 3 & 53 & 1.5 & 14 & 14 & 0 & 0 \\
\hline $\begin{array}{l}\text { Orman et al., } \\
2013 \text { [42] }\end{array}$ & 112 & 64.1 & 79.5 & 4 & 107 & 1.1 & 8 & 3 & 2 & 3 \\
\hline $\begin{array}{l}\text { Phoa et al., } \\
2013 \text { [33] }\end{array}$ & 55 & 65 & 81.8 & 5 & 54 & 5.1 & 25 & 22 & 1 & 2 \\
\hline $\begin{array}{l}\text { Shue et al., } \\
2013 \text { [47] }\end{array}$ & 42 & 61 & 71.4 & 3.5 & 42 & 1.17 & 11 & 11 & 0 & 0 \\
\hline $\begin{array}{l}\text { Pasricha et al., } \\
2014 \text { [32] }\end{array}$ & 1634 & 61.7 & 74 & 4 & NR & NR & 334 & 269 & 52 & 13 \\
\hline $\begin{array}{l}\text { Strauss et al., } \\
2014 \text { [61] }\end{array}$ & 36 & 64 & 72.2 & 3.5 & 32 & 2 & 9 & 5 & 3 & 1 \\
\hline $\begin{array}{l}\text { Agoston et al., } \\
2015 \text { [40] }\end{array}$ & 78 & 67.1 & 75.6 & 4.1 & 67 & 2.2 & - & - & - & 6 \\
\hline $\begin{array}{l}\text { Cotton et al., } \\
2015 \text { [43] }\end{array}$ & 198 & 69.8 & 70.7 & 4.7 & 198 & 3 & 35 & 22 & 7 & 6 \\
\hline $\begin{array}{l}\text { Lada et al., } \\
2014 \text { [62] }\end{array}$ & 57 & 66.2 & 87.7 & 5.1 & 57 & 2.95 & 16 & 4 & 12 & 0 \\
\hline
\end{tabular}


- Table 2 (Continuation)

\begin{tabular}{|c|c|c|c|c|c|c|c|c|c|c|}
\hline Study & $\begin{array}{l}\text { Total } \\
\text { no. }\end{array}$ & $\begin{array}{l}\text { Mean } \\
\text { age, } \\
\text { years }\end{array}$ & $\begin{array}{l}\% \\
\text { Male }\end{array}$ & $\begin{array}{l}\text { Mean } \\
\text { BE } \\
\text { length, } \\
\mathrm{cm}\end{array}$ & $\begin{array}{l}\text { Total no. } \\
\text { of pts } \\
\text { followed }\end{array}$ & $\begin{array}{l}\text { Mean } \\
\text { follow- } \\
\text { up } \\
\text { length, } \\
\text { years }\end{array}$ & $\begin{array}{l}\text { Total } \\
\text { recurren- } \\
\text { ces }\end{array}$ & $\begin{array}{l}\text { IM recur- } \\
\text { rences }\end{array}$ & $\begin{array}{l}\text { Dysplasia } \\
\text { recurren- } \\
\text { ces }\end{array}$ & $\begin{array}{l}\text { EAC recur- } \\
\text { rences }\end{array}$ \\
\hline $\begin{array}{l}\text { Le Page et al., } \\
2016 \text { [63] }\end{array}$ & 50 & 67 & 72 & 5 & 45 & 1.75 & - & - & - & 2 \\
\hline $\begin{array}{l}\text { Phoa et al., } \\
2015 \text { [64] }\end{array}$ & 132 & 65 & 81 & 6 & 121 & 2.25 & 10 & 5 & 3 & 2 \\
\hline $\begin{array}{l}\text { Small et al., } \\
2015 \text { [44] }\end{array}$ & 197 & 67.9 & 84.8 & 2.4 & 197 & 2.87 & 81 & 64 & 15 & 2 \\
\hline \multicolumn{11}{|l|}{ SRER group } \\
\hline $\begin{array}{l}\text { Giovannini } \\
\text { et al., } 2004 \text { [65] }\end{array}$ & 21 & 63 & 52.4 & 3.5 & 18 & 1.5 & 2 & 0 & 2 & 0 \\
\hline $\begin{array}{l}\text { Larghi et al., } \\
2007 \text { [66] }\end{array}$ & 24 & 64.1 & 83.3 & 2.5 & 24 & 2.3 & 3 & 2 & 0 & 1 \\
\hline $\begin{array}{l}\text { Lopes et al., } \\
2007 \text { [67] }\end{array}$ & 41 & 65.8 & 83.4 & 4.9 & 41 & 2.6 & 11 & 10 & 0 & 1 \\
\hline $\begin{array}{l}\text { Chennat et al., } \\
2009 \text { [68] }\end{array}$ & 49 & 67 & 75.5 & 3.2 & 32 & 1.9 & 1 & 1 & 0 & 0 \\
\hline $\begin{array}{l}\text { Brahmania } \\
\text { et al., } 2010 \text { [69] }\end{array}$ & 22 & 67 & 100 & 5.5 & 18 & 2 & 4 & 0 & 4 & 0 \\
\hline $\begin{array}{l}\text { Moss et al., } \\
2010[70]\end{array}$ & 75 & 68 & 78.7 & 3.6 & 33 & 2.6 & 0 & 0 & 0 & 0 \\
\hline $\begin{array}{l}\text { Chung et al., } \\
2011 \text { [26] }\end{array}$ & 77 & 65 & 83.1 & 2 & 73 & 1.4 & 5 & 2 & 3 & 0 \\
\hline $\begin{array}{l}\text { Gerke et al., } \\
2011 \text { [25] }\end{array}$ & 41 & 67 & 78 & 3.2 & 32 & 2.1 & 3 & 3 & 0 & 0 \\
\hline $\begin{array}{l}\text { van Vilsteren et } \\
\text { al., } 2011[34]^{1}\end{array}$ & 25 & 68 & 84 & 4 & 25 & 2.1 & 3 & 2 & 0 & 1 \\
\hline $\begin{array}{l}\text { Anders et al., } \\
2014 \text { [27] }\end{array}$ & 90 & 63 & 91.1 & NR & 81 & 5.4 & 37 & 32 & 2 & 3 \\
\hline $\begin{array}{l}\text { Conio et al., } \\
2014 \text { [71] }\end{array}$ & 47 & 65 & 91.5 & 3 & 43 & 1.5 & 2 & 0 & 1 & 1 \\
\hline $\begin{array}{l}\text { Konda et al., } \\
2014 \text { [28] }\end{array}$ & 86 & 67.5 & NR & 3.6 & 74 & 2.8 & 15 & 7 & 7 & 1 \\
\hline $\begin{array}{l}\text { Belghazi et al., } \\
2016 \text { [72] }\end{array}$ & 73 & 64 & 87.6 & 3 & 73 & 6.33 & 17 & 16 & 0 & 1 \\
\hline \multicolumn{11}{|c|}{ Both SRER and RFA combined } \\
\hline $\begin{array}{l}\text { Wani et al., } \\
2016 \text { [51] }\end{array}$ & 542 & 67 & 79.5 & 5.4 & 446 & 2.75 & 127 & 85 & 42 & 0 \\
\hline $\begin{array}{l}\text { Waxman et al., } \\
2016 \text { [73] }\end{array}$ & 152 & 64.9 & 90.1 & 5 & 129 & 3.53 & 48 & 34 & 14 & \\
\hline
\end{tabular}


Incidence of total recurrence across all studies

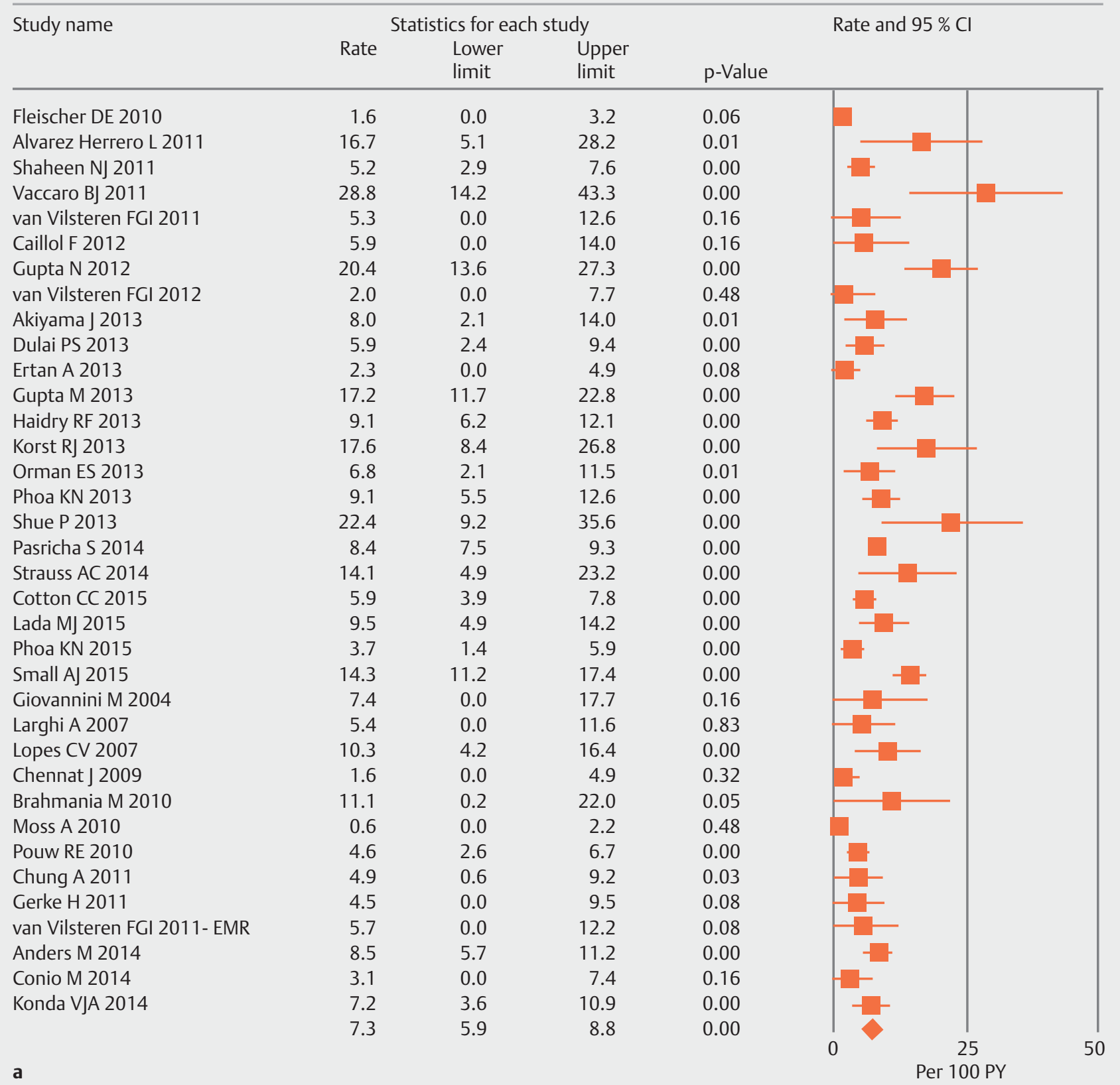

- Fig. 2a Overall pooled incidence of any recurrence (intestinal metaplasia or dysplasia) after achieving complete eradication of intestinal metaplasia following endoscopic eradication therapy using stepwise complete endoscopic resection or radiofrequency ablation with or without focal endoscopic mucosal resection.

patients with HGD, but not EAC, had an increased risk of developing recurrent dysplasia, while older age and non-Caucasian race had a higher risk of recurrent dysplasia [44]. Vacarro et al. [36] found that BE length was significantly associated with increased recurrence $(P=0.03)$. Other risk factors identified by Shue et al. that increased the risk of IM recurrence included smoking (39.3\% vs $0 \%$ among those with and without recurrence, $P=0.006$ ) and mean $B M I$ (25.3 vs 29.8 among those with and without recurrence, $P=0.002$ ) [47]. Only one study focused on recurrence post SRER and reported $B E$ length as a pre- dictor for IM and dysplasia on multivariable regression analysis [OR $2.73(95 \% \mathrm{Cl} 1.01-7.38)$ ] [27].

\section{Publication bias}

Using Egger's regression intercept, the two-tailed $P$ value of the publication bias for total recurrence and IM recurrence among all studies was 0.14 and 0.46 , respectively, suggesting the lack of publication bias. The funnel plot for total recurrence is shown in $>$ Fig. 3. The $P$ value for early neoplasia (EN) recur- 
Incidence of IM recurrence across all studies

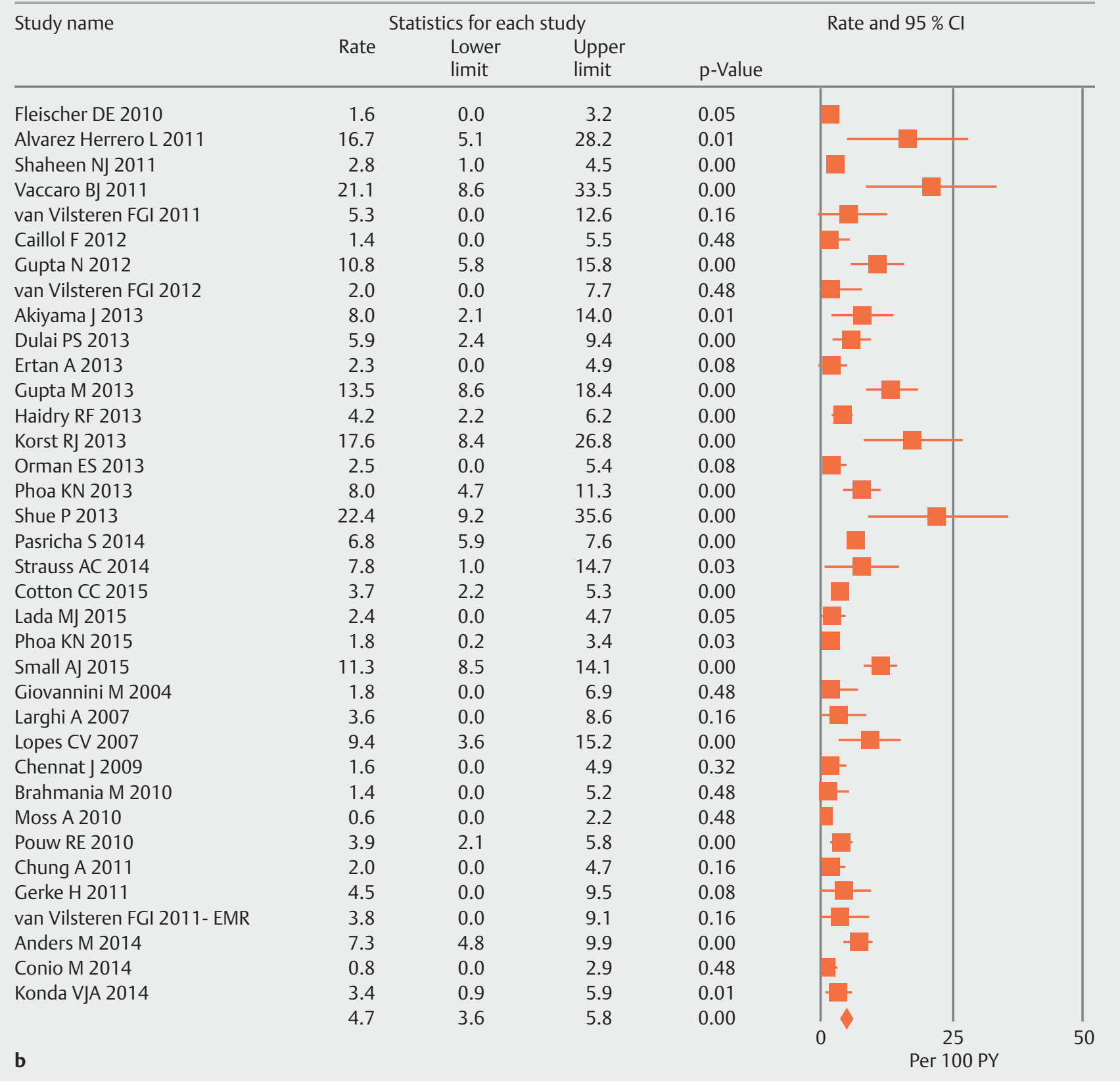

- Fig. 2b Overall pooled incidence of intestinal metaplasia after achieving complete eradication of intestinal metaplasia following endoscopic eradication therapy using stepwise complete endoscopic resection or radiofrequency ablation with or without focal endoscopic mucosal resection.

rence was $<0.001$, suggesting the presence of a publication bias in this subgroup.

\section{Discussion}

EET in BE patients at highest risk for progression to invasive cancer (mucosal EAC, HGD and LGD) is a well-established and effective treatment strategy that minimizes cancer risk and reduces the morbidity and mortality associated with EAC. This practice has now been endorsed by gastrointestinal societal guidelines and has replaced esophagectomy as the standard of care for HGD and EAC confined to the mucosa $[8,48,49]$. While the effectiveness of EET has been demonstrated in randomized controlled trials and large observational studies, variable rates of recurrence of IM and dysplasia have been reported postEET. A reliable estimate of the risk of recurrence after achieving CE-IM is critical to establish surveillance guidelines (duration and frequency of surveillance endoscopy), determine cost-effectiveness of EET, and ultimately educate physicians and patients before embarking on EET. 
Incidence of dysplasia recurrewnce across all studies

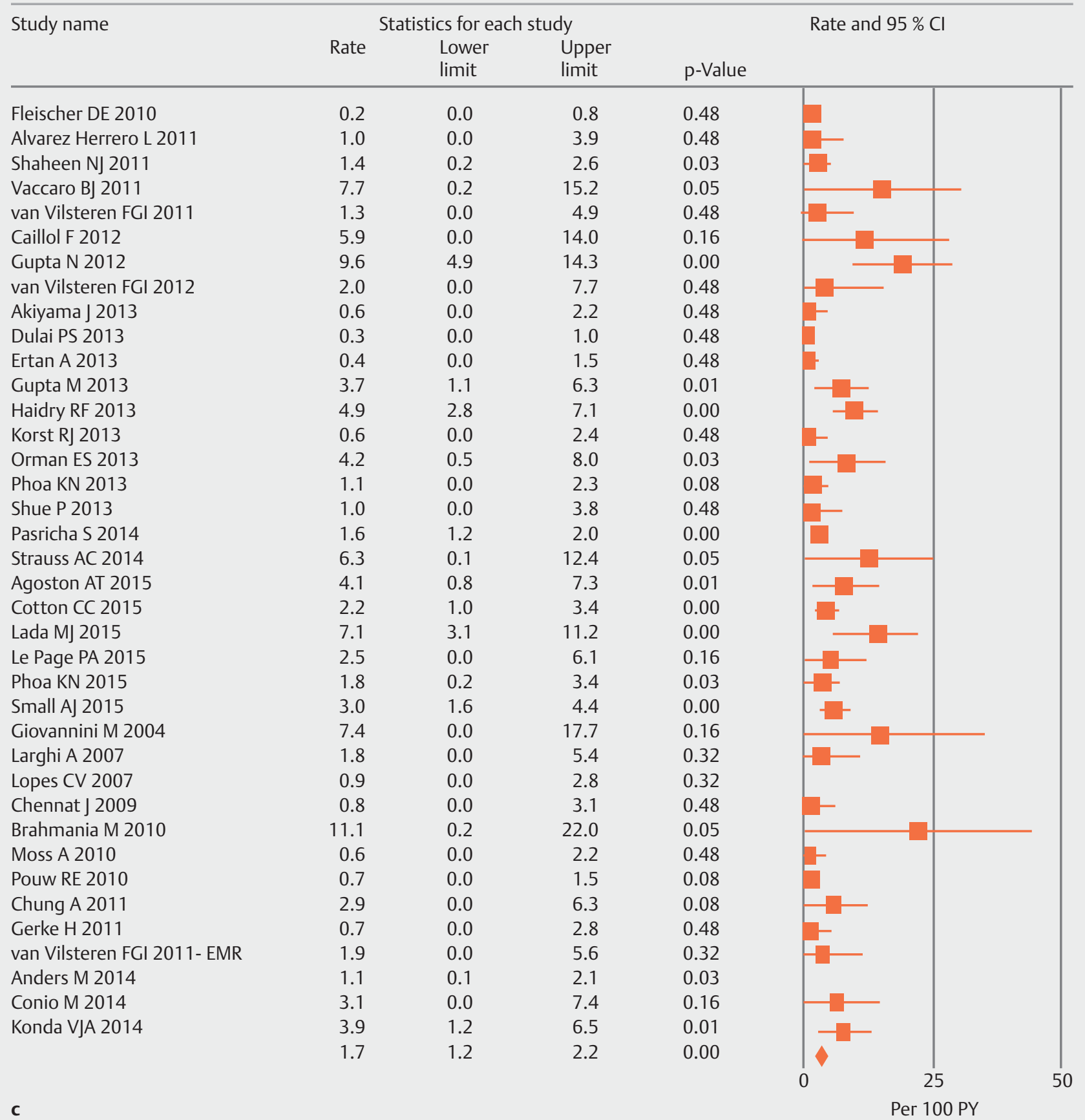

- Fig.2c Overall pooled incidence of early neoplasia (EN) after achieving complete eradication of intestinal metaplasia following endoscopic eradication therapy using stepwise complete endoscopic resection or radiofrequency ablation with or without focal endoscopic mucosal resection.

The results of this systematic review and meta-analysis demonstrate a pooled incidence rate for any recurrence, IM, and dysplasia of $7.5 \% / 100$ PY $(95 \% \mathrm{Cl} 6.1-9), 4.8 \% / 100$ PY $(95 \% \mathrm{Cl}$ $3.8-5.9)$, and $2.0 \% / 100$ PY $(95 \% \mathrm{Cl} 1.5-2.5)$, respectively using EET treatment modalities (RFA with or without focal EMR and SRER). In addition, the vast majority of recurrences were amenable to repeat EET. In a recent systematic review and meta-analysis, Krishnamoorthi and colleagues also reported the risk of recurrence of IM, dysplasia, and HGD/EAC in patients undergoing RFA and recurrence rates after use of all endoscopic modalities [50]. While the primary results were similar, the current study focused primarily on contemporary EET therapies and also compared recurrence rates between RFA and SRER. Similar to the above study, the current study with updated 


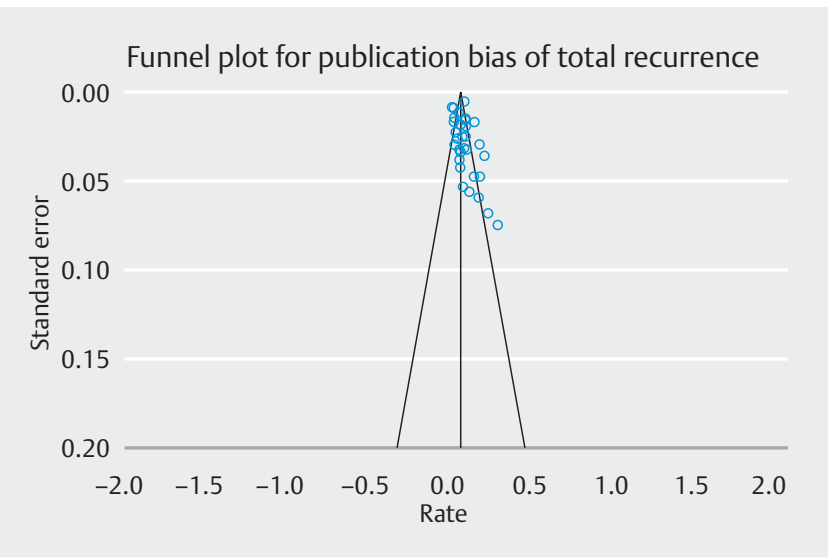

- Fig. 3 Funnel plot for publication bias on the incidence of total recurrence among all studies.

search results noted substantial heterogeneity. Another study that provided a recurrence estimate was a study by Orman et al. that determined the efficacy and durability of RFA for patients with dysplastic and non-dysplastic BE. That study reported the proportion of patients with recurrence of IM after successful CE-IM with RFA therapy and quoted a point estimate of $13 \%$ (95\% Cl 9-18\%) [31]. However, reporting recurrence rate per patient-year of follow-up (accounting for follow-up duration) is a more stable and meaningful outcome.

The results of this study raise the important issue with regard to the optimal EET strategy (SRER vs RFA with or without EMR) with regard to recurrence of IM and dysplasia. Patients in the RFA group had higher overall and IM recurrence rates compared to patients treated with SRER; however, there was no difference between the two groups with regard to recurrence of dysplasia. The authors acknowledge that any direct comparison between RFA and SRER is biased as only one study directly compared these two strategies in a randomized controlled trial. We emphasize that any comparison between RFA and SRER is indirect based on the rates of recurrence in each arm obtained by combining observational studies. Although several studies have confirmed the effectiveness and durability of SRER, this treatment approach is associated with a high stricture rate [27-29]. In a multicenter randomized controlled trial compar- ing these two treatment approaches, a higher stricture rate was reported in patients treated with SRER compared to patients treated with focal EMR followed by RFA ( $88 \%$ vs $14 \%, P<$ 0.001 ) with no difference in efficacy indicators of CE-IM and CE-D [34]. This justifies the current strategy of focal EMR for visible lesions followed by ablation of the residual Barrett's segment for optimal treatment of BE related neoplasia [8, 48, 49]. In addition, the studies evaluating SRER limited the length of $\mathrm{BE}$ in the included patients and, given that many studies identify BE length as a risk factor for recurrence, the lower recurrence rate (overall and IM) in the SRER group may be confounded by BE length. Whether there is a subgroup of BE-related neoplasia patients (multifocal disease, diffuse nodularity) more likely to benefit from SRER needs to be evaluated in future studies. Improvement in prevention of stricture formation post-SRER is also required before this treatment strategy can be recommended as a primary treatment modality.

This systematic review highlights the variable rates of recurrence reported in the literature, an important take-home message of this study. The purported factors for this include study design (prospective vs retrospective), participating centers (tertiary care vs community practices), sample size, variability in study inclusion criteria (histologic grades of BE), treatment and surveillance protocol, duration of follow-up, and definitions of CE-IM and recurrence (whether IM at or just below the neo-squamocolumnar junction is included in the numerator of patients with recurrences). Although some studies reported on subsquamous IM recurrence and recurrence of the cardia, the significance of these findings was unclear. With the low number of studies focusing on these outcomes, this was not a focus of the manuscript. Several factors in the subgroup analyses were associated with a significant difference in incidence rates; however, none completely account for the heterogeneity noted in this meta-analysis. For example, in the RFA group, prospective studies were associated with a lower incidence of total, IM, and dysplasia recurrence rates compared to retrospective studies and a higher dysplasia recurrence rate was reported in trials conducted at tertiary care centers. This may be related to inherent limitations associated with retrospective and multicenter studies (lack of standardized definitions, treatment and surveillance protocol). Contrary to previous studies, recurrence of dysplasia was higher in $\mathrm{BE}$ patients with length $<3 \mathrm{~cm}$. The rea-

- Table 3 Incidence of recurrence after achieving CE-IM following EET.

\begin{tabular}{|c|c|c|c|c|c|c|}
\hline & \multicolumn{2}{|c|}{ Total recurrence } & \multicolumn{2}{|c|}{ IM recurrence } & \multicolumn{2}{|c|}{ Dysplasia recurrence } \\
\hline & $\begin{array}{l}\text { No. of } \\
\text { studies }\end{array}$ & $\begin{array}{l}\text { Incidence per } 100 \mathrm{PY} \\
(95 \% \mathrm{Cl})\end{array}$ & $\begin{array}{l}\text { No. of } \\
\text { studies }\end{array}$ & $\begin{array}{l}\text { Incidence per } 100 \mathrm{PY} \\
(95 \% \mathrm{Cl})\end{array}$ & $\begin{array}{l}\text { No. of } \\
\text { studies }\end{array}$ & $\begin{array}{l}\text { Incidence per } 100 \mathrm{PY} \\
(95 \% \mathrm{CI})\end{array}$ \\
\hline Overall & $36^{1}$ & $6.8(5.4-8.1)$ & $35^{1}$ & $4.4(3.4-5.4)$ & $38^{1}$ & $1.6(1.1-2.1)$ \\
\hline RFA & 23 & $8.6(6.7-10.5)$ & 23 & $5.8(4.3-7.3)$ & 25 & $1.9(1.3-2.5)$ \\
\hline SRER & 13 & $4.9(3-6.8)$ & 12 & $3.3(2-4.7)$ & 13 & $1.2(0.4-1.9)$ \\
\hline \multicolumn{7}{|c|}{$\begin{array}{l}\text { CE-IM = complete eradication of intestinal metaplasia, EET = endoscopic eradication therapy, RFA = radiofrequency ablation, SRER=stepwise complete endoscopic } \\
\text { resection, IM = intestinal metaplasia, PY= patient-years; Dysplasia recurrence includes dysplasia and EAC. } \\
{ }^{1} \text { One study compared RFA to SRER. Its data is separated into each group, making the overall number of studies one less than the total RFA and SRER studies com } \\
\text { bined. }\end{array}$} \\
\hline
\end{tabular}


- Table 4 Treatment of recurrent intestinal metaplasia and dysplasia.

\begin{tabular}{|c|c|c|c|c|c|}
\hline Study & $\begin{array}{l}\text { Total } \\
\text { recurrences }\end{array}$ & $\begin{array}{l}\text { IM } \\
\text { recurrences }\end{array}$ & $\begin{array}{l}\text { Dysplasia } \\
\text { recurrences }\end{array}$ & $\begin{array}{l}\text { EAC } \\
\text { recurrences }\end{array}$ & Management of recurrence \\
\hline Fleischer et al., 2010 [52] & 4 & 4 & 0 & 0 & NR \\
\hline Alvarez Herrero et al., 2011 [53] & 8 & 8 & 0 & 0 & NR \\
\hline Shaheen et al., 2011 [30] & 19 & 14 & 3 & 2 & RFA \\
\hline Vaccaro et al., 2011 [36] & 15 & 11 & 4 & 0 & RFA, EMR \\
\hline van Vilsteren et al., 2011 [34] ${ }^{1}$ & 2 & 2 & 0 & 0 & NR \\
\hline Caillol et al., 2012 [54] & 2 & 0 & 0 & 2 & NR \\
\hline Gupta et al., 2012 [55] & 34 & 18 & 16 & 0 & APC/RFA, EMR \\
\hline van Vilsteren et al., 2012 [56] & 0 & 0 & 0 & 0 & No recurrence \\
\hline Akiyama et al., 2013 [45] & 7 & 7 & 0 & 0 & NR \\
\hline Dulai et al., 2013 [57] & 11 & 11 & 0 & 0 & RFA \\
\hline Ertan et al., 2013 [58] & 3 & 3 & 0 & 0 & All three underwent surgery \\
\hline Gupta et al., 2013 [35] & 37 & 29 & 8 & 0 & NR \\
\hline Haidry et al., 2013 [59] & 37 & 17 & 16 & 4 & NR \\
\hline Korst et al., 2013 [60] & 14 & 14 & 0 & 0 & RFA \\
\hline Orman et al., 2013 [42] & 8 & 3 & 2 & 3 & RFA \\
\hline Phoa et al., 2013 [33] & 25 & 22 & 1 & 2 & NR \\
\hline Shue et al., 2013 [47] & 11 & 11 & 0 & 0 & NR \\
\hline Pasricha et al., 2014 [32] & 334 & 269 & 52 & 13 & NR \\
\hline Strauss et al., 2014 [61] & 9 & 5 & 3 & 1 & RFA \\
\hline Agoston et al., 2015 [40] & - & - & - & 6 & $\begin{array}{l}\text { Unclear as primary failure not } \\
\text { differentiated from recurrences }\end{array}$ \\
\hline Cotton et al., 2015 [43] & 35 & 22 & 7 & 6 & NR \\
\hline Lada et al., 2014 [62] & 16 & 4 & 12 & 0 & NR \\
\hline Le Page PA 2016 [63] & - & - & - & 2 & APC/RFA, EMR \\
\hline Phoa et al., 2015 [64] & 10 & 5 & 3 & 2 & APC, EMR \\
\hline Small et al., 2015 [44] & 81 & 64 & 15 & 2 & $\begin{array}{l}\text { Treated endoscopically but did } \\
\text { not specify how }\end{array}$ \\
\hline Giovannini et al., 2004 [65] & 2 & 0 & 2 & 0 & NR \\
\hline Larghi et al., 2007 [66] & 3 & 2 & 0 & 1 & NR \\
\hline Lopes et al., 2007 [67] & 11 & 10 & 0 & 1 & NR \\
\hline Chennat et al., 2009 [68] & 1 & 1 & 0 & 0 & NR \\
\hline Brahmania et al., 2010 [69] & 4 & 0 & 4 & 0 & $\begin{array}{l}\text { All recurrences were LGD and } \\
\text { under surveillance }\end{array}$ \\
\hline Moss et al., 2010 [70] & 0 & 0 & 0 & 0 & No recurrence \\
\hline Chung et al., 2011 [26] & 5 & 2 & 3 & 0 & EMR \\
\hline Gerke et al., 2011 [25] & 3 & 3 & 0 & 0 & RFA, EMR \\
\hline van Vilsteren et al., 2011 [34] ${ }^{1}$ & 3 & 2 & 0 & 1 & NR \\
\hline Anders et al., 2014 [27] & 37 & 32 & 2 & 3 & APC, EMR \\
\hline Conio et al., 2014 [71] & 2 & 0 & 1 & 1 & NR \\
\hline Konda et al., 2014 [28] & 15 & 7 & 7 & 1 & EMR \\
\hline
\end{tabular}


Table 4 (Continuation)

\begin{tabular}{|l|l|l|l|l|l|}
\hline Study & $\begin{array}{l}\text { Total } \\
\text { recurrences }\end{array}$ & $\begin{array}{l}\text { IM } \\
\text { recurrences }\end{array}$ & $\begin{array}{l}\text { Dysplasia } \\
\text { recurrences }\end{array}$ & $\begin{array}{l}\text { EAC } \\
\text { recurrences }\end{array}$ & Management of recurrence \\
\hline Belghazi et al., 2016 [72] & 17 & 16 & 0 & 1 & Esophagectomy for T1b cancer \\
\hline Wani et al., 2016 [51] & 127 & 85 & 42 & NR \\
\hline Waxman et al., 2016 [73] & 48 & 34 & 14 & NR \\
\hline $\begin{array}{l}\text { NR =not reported, RFA= radiofrequency ablation, EMR = endoscopic mucosal resection, APC=argon plasma coagulation, LGD = low grade dysplasia. } \\
\text { 1 Randomized trial of RFA vs SRER, therefore the patient data was split into the corresponding groups. }\end{array}$
\end{tabular}

son for this finding is unclear. Subgroup analyses provided no evidence that overall recurrence rates are higher in patients with $B E$ related neoplasia compared to those with non-dysplastic BE. Consistent with the results from the US RFA Registry [32], there was no difference in recurrence rates based on the definition of CE-IM (CE-IM defined by one or two successive negative endoscopies).

There are limitations of this systematic review and meta-analysis that merit discussion. One inherent limitation of the study design is the quality of the individual studies. The majority of the studies that met the inclusion criteria were of low to moderate quality, with only three studies meeting the criteria for high quality. This is predominantly because recurrence after EET was often a secondary analysis rather than the primary end point of the study. Another major limitation is the substantial heterogeneity noted in the assessment of recurrence rates. This may be related to patient characteristics, significant variability in the baseline pathology of included patients (proportion of included patients with HGD/early EAC), endoscopic surveillance intervals, surveillance biopsy protocols, confirmation by a dedicated gastrointestinal pathologist, and variability in definition of recurrence between each study. This was addressed by using strict inclusion and exclusion criteria and a priori subgroup analyses to explore sources of heterogeneity. However, the authors acknowledge that the substantial heterogeneity tempers the confidence level associated with the estimates of recurrence reported in this study and the precision of these estimates may change as data from large prospective multicenter trials become available.

The authors acknowledge that it would be ideal to conduct a time-to-event analysis accounting for individual patient data and patients lost to follow-up. However, these data were reported in few studies thus precluding this analysis. This study also highlights the fact that only a limited number of studies report on the risk factors associated with recurrence, and they comprise only a small number of overall recurrences, limiting our ability to perform multivariable regression analysis. Hence, only a descriptive analysis of risk factors was provided in this study. The associations between the presence of erosive esophagitis, older age, non-Caucasian race, pretreatment BE length, and recurrence of IM and dysplasia need to be explored and confirmed in future studies. The generalizability of these estimates is limited by the fact that most of the included studies were conducted at tertiary care centers. Future studies need to clarify if differences in technique and protocols, for instance, the routine performance of circumferential RFA at the gastroesophageal junction (location of most recurrences) at each RFA session, regardless of the presence of visible $B E$, impact the durability of EET. Similarly, differences in acid suppressive regimens need to be correlated with the likelihood of recurrence of IM and dysplasia. This study did not address the durability of other ablative techniques such as argon plasma coagulation but focused on the most widely used techniques.

Finally, temporality of recurrence after achieving CE-IM cannot be established with the available data in published reports. Although surveillance post-CE-IM is uniformly recommended, surveillance intervals suggested by current guidelines [8] are largely driven by expert opinion and low quality evidence. Future prospective studies need to provide guidance on current surveillance strategies post CE-IM. The strengths of this study include the use of strict study definitions and inclusion criteria, and provision of point estimates for incidence of recurrence for IM and dysplasia per patient-year for contemporary EET modalities.

In conclusion, this systematic review highlights the variable rates of recurrence reported in the literature. The results of this meta-analysis report the incidence rates of recurrence of IM and dysplasia after successful CE-IM in patients with BE postEET. The vast majority of recurrences were without dysplasia and could be managed with repeat EET, adding credence to the current strategy of EET for management of BE-related neoplasia. This study emphasizes the importance of routine longterm endoscopic surveillance following EET and highlights the need to discuss these recurrence estimates with patients before embarking on EET. Future prospective studies that use standardized definitions for study end points and focus on recurrence risk as the primary outcome are required to more precisely define the annual recurrence risk and the predictors associated with recurrence. This will allow for evidence-based recommendations with regard to surveillance endoscopic and biopsy protocols after successful EET of BE-related neoplasia patients with the goal of stopping or reducing the frequency of surveillance in low risk individuals and enrolling high risk patients in an intensive surveillance protocol. 
- Table 5 Subgroup analyses on the incidence of any recurrence and recurrent IM and EN after RFA.

\begin{tabular}{|c|c|c|c|c|c|c|c|c|c|c|}
\hline & $\begin{array}{l}\text { No. of } \\
\text { stud- } \\
\text { ies }\end{array}$ & $\begin{array}{l}\text { Total recurrence } \\
\text { [incidence per } \\
100 \mathrm{PY}(95 \% \mathrm{Cl}) \text { ] }\end{array}$ & $I^{2}$ & $\begin{array}{l}P \text { val- } \\
\text { ue }\end{array}$ & $\begin{array}{l}\text { IM recurrence } \\
\text { [incidence per } \\
100 \text { PY ( } 95 \% \\
\mathrm{CI}) \text { ] }\end{array}$ & $P^{2}$ & $\begin{array}{l}P \text { val- } \\
\text { ue }\end{array}$ & $\begin{array}{l}\text { EN recur- } \\
\text { rence [inci- } \\
\text { dence per } \\
100 \text { PY ( } 95 \% \\
\text { CI)] }\end{array}$ & $P^{2}$ & $\begin{array}{l}P \text { val- } \\
\text { ue }\end{array}$ \\
\hline \multicolumn{4}{|l|}{ Manuscript type } & 0.11 & & & 0.04 & & & 0.55 \\
\hline - Full text & 20 & $7.9(6-9.8)$ & 77.20 & & $5.3(3.8-6.8)$ & 47.01 & & $1.9(1.2-2.5)$ & 70.54 & \\
\hline - Abstract & 3 & $16.1(6.4-25.9)$ & 86.30 & & $11.4(5.8-17)$ & 85.24 & & $3.1(0-7.3)$ & 84.39 & \\
\hline \multicolumn{4}{|l|}{ Study outlook } & 0.001 & & & $<0.001$ & & & 0.01 \\
\hline - Prospective & 5 & $4.1(1.6-6.6)$ & 68.15 & & $2.4(0.9-4)$ & 49.18 & & $0.9(0.1-1.7)$ & 34.15 & \\
\hline - Retrospective & 18 & $9.7(7.6-11.7)$ & 80.53 & & $6.5(4.8-8.2)$ & 81.13 & & $2.3(1.5-3)$ & 71.08 & \\
\hline Study design & & & & 0.62 & & & 0.80 & & & 0.19 \\
\hline - Case series & 5 & $8.1(3.3-12.9)$ & 88.48 & & $7.5(2.7-12.6)$ & 85.75 & & $1.2(0.4-2)$ & 0 & \\
\hline - Cohort & 17 & $9(6.8-11.3)$ & 77.75 & & $5.7(4-7.3)$ & 84.18 & & $2.2(1.5-3)$ & 78.29 & \\
\hline $\begin{array}{l}\text { - Randomized } \\
\text { control }\end{array}$ & 1 & $5.3(0-12)$ & 0 & & $5.3(0-12.6)$ & 0 & & $1.3(0-0.5$ & 0 & \\
\hline \multicolumn{4}{|l|}{ Setting } & 0.94 & & & 0.80 & & & 0.29 \\
\hline - Multicenter & 10 & $8.8(5.9-11.8)$ & 90.97 & & $5.7(3.5-7.9)$ & 88.68 & & $2.3(1.3-3.3)$ & 79.89 & \\
\hline - Single center & 13 & $8.7(5.9-11.4)$ & 79.77 & & $6.1(3.8-8.4)$ & 80.76 & & $1.6(0.3-2.5)$ & 61.22 & \\
\hline \multicolumn{4}{|l|}{ Hospital type } & 0.24 & & & 0.68 & & & 0.03 \\
\hline - Tertiary only & 20 & $9.4(7.1-11.6)$ & 96.32 & & $6(4.3-7.7)$ & 94.01 & & $2.4(1.6-3.1)$ & 67.36 & \\
\hline $\begin{array}{l}\text { - Included } \\
\text { community }\end{array}$ & 3 & $5.8(0.3-11.3)$ & 81.82 & & $5.1(0.8-9.4)$ & 80.83 & & $0.8(0-2)$ & 88.57 & \\
\hline \multicolumn{4}{|l|}{ Location } & 0.13 & & & 0.13 & & & 0.65 \\
\hline - USA & 16 & $9.6(7.2-12)$ & 89.43 & & $6.5(4.6-7.1)$ & 86.98 & & $1.8(1.1-2.6)$ & 77.3 & \\
\hline - Non-USA & 7 & $6.7(3.8-9.5)$ & 63.38 & & $4.2(1.8-6.5)$ & 66.58 & & $2.2(1-3.3)$ & 34.87 & \\
\hline \multicolumn{4}{|l|}{ Quality } & 0.20 & & & 0.51 & & & 0.06 \\
\hline - Low & 13 & $8.6(5.9-11.4)$ & 78.19 & & $5.5(3.5-7.6)$ & 75.08 & & $2(1-3)$ & 69.88 & \\
\hline - Moderate & 7 & $6.9(3.7-10.1)$ & 91.12 & & $4.8(2.3-7.2)$ & 88.56 & & $1.5(0.6-2.4)$ & 76.19 & \\
\hline " High & 3 & $12.7(7.2-18.3)$ & 79.1 & & $9(2.2-15.7)$ & 91.68 & & $3.3(2.1-4.5)$ & 0 & \\
\hline \multicolumn{4}{|c|}{ Non-dysplastic BE included } & 0.85 & & & 0.57 & & & 0.02 \\
\hline - No & 12 & $8.9(6-11.8)$ & 82.11 & & $5.5(3.4-7.5)$ & 78.5 & & $2.9(1.7-4)$ & 69.42 & \\
\hline - Yes & 11 & $8.5(5.7-11.3)$ & 89.51 & & $6.4(4-8.7)$ & 88.85 & & $1.2(0.5-1.9)$ & 71.27 & \\
\hline \multicolumn{4}{|c|}{ Number of EGDs negative for IM and $E N$ required to define $C E$} & 0.78 & & & 0.51 & & & 0.48 \\
\hline .1 & 14 & $7.8(5.9-9.7)$ & 78.5 & & $5.7(3.8-7.5)$ & 84.54 & & $1.9(1.2-2.5)$ & 59.84 & \\
\hline - 2 & 3 & $9.2(0-18.5)$ & 95.37 & & $5.1(0.4-9.9)$ & 90.24 & & $3.3(0-7.2)$ & 88.57 & \\
\hline \multicolumn{4}{|l|}{ Cardia biopsied } & 0.61 & & & 0.48 & & & 0.67 \\
\hline - No & 8 & $6.6(3.7-9.5)$ & 91.54 & & $4.5(2.4-6.6)$ & 88.74 & & $1.4(0.5-2.2)$ & 82.98 & \\
\hline - Yes & 9 & $7.7(4.8-10.6)$ & 71.1 & & $5.7(3.1-8.3)$ & 76.2 & & $1.6(1-2.3)$ & & \\
\hline \multicolumn{4}{|c|}{ Surveillance biopsy protocol, cm } & 0.08 & & & 0.04 & & & 0.99 \\
\hline - 1 & 8 & $5.5(3.2-7.9)$ & 89.53 & & $3.5(1.7-5.3)$ & 87.51 & & $1.4(0.6-2.2)$ & 80.72 & \\
\hline . $>1$ & 9 & $9.8(5.6-14.1)$ & 78.33 & & $8.1(4.1-12.1)$ & 83.76 & & $1.4(0.7-2.1)$ & & \\
\hline \multicolumn{4}{|l|}{ BE length $>3 \mathrm{~cm}$} & 0.04 & & & 0.002 & & & 0.69 \\
\hline - No & 3 & $17.4(11-23.9)$ & 48.66 & & $14.5(8.7-20.3)$ & 45.37 & & $2.4(0-4.8)$ & 68.89 & \\
\hline - Yes & 20 & $7.6(5.7-9.4)$ & 84.47 & & $4.9(3.5-6.7)$ & 82.16 & & $1.9(1.2-2.5)$ & 72.03 & \\
\hline
\end{tabular}

$E G D=$ esophagogastroduodenoscopy, IM = intestinal metaplasia, $E N=$ early neoplasia, $C E=$ complete eradication, $B E=$ Barrett's esophagus, PY= patient-years, $R F A=$ radiofrequency ablation. 
Table 6 Subgroup analyses on the incidence of recurrent IM and EN after SRER.

\begin{tabular}{|c|c|c|c|c|c|c|c|c|c|c|}
\hline & $\begin{array}{l}\text { No. } \\
\text { of } \\
\text { stud- } \\
\text { ies }\end{array}$ & $\begin{array}{l}\text { Total recurrence } \\
\text { [incidence per } \\
100 \text { PY }(95 \% \mathrm{Cl}) \text { ] }\end{array}$ & $P^{2}$ & $\begin{array}{l}P \text { val- } \\
\text { ue }\end{array}$ & $\begin{array}{l}\text { IM recurrence } \\
\text { [incidence per } \\
100 \mathrm{PY}(95 \% \\
\mathrm{CI}) \text { ] }\end{array}$ & $P^{2}$ & $\begin{array}{l}P \text { val- } \\
\text { ue }\end{array}$ & $\begin{array}{l}\text { EN recurrence } \\
\text { [incidence per } \\
100 \text { PY ( } 95 \% \\
\text { CI)] }\end{array}$ & $P^{2}$ & $\begin{array}{l}P \text { val- } \\
\text { ue }\end{array}$ \\
\hline \multicolumn{4}{|l|}{ Manuscript type } & 0.3 & & & 0.9 & & & 0.004 \\
\hline $\begin{array}{l}\text { - Full text } \\
\text { - Abstract }\end{array}$ & $\begin{array}{r}12 \\
1\end{array}$ & $\begin{array}{l}5.2(2.9-7.5) \\
3.7(1.9-5.4)\end{array}$ & $\begin{array}{r}72 \\
0\end{array}$ & & $\begin{array}{l}3.3(1.7-4.9) \\
3.5(1.8-5.2)\end{array}$ & $\begin{array}{c}55.85 \\
0\end{array}$ & & $\begin{array}{l}1.3(0.7-2) \\
0.2(0-0.6)\end{array}$ & $\begin{array}{l}2.23 \\
0\end{array}$ & \\
\hline \multicolumn{4}{|l|}{ Study outlook } & 0.22 & & & 0.45 & & & 0.95 \\
\hline $\begin{array}{l}\text { - Prospective } \\
\text { - Retrospective }\end{array}$ & $\begin{array}{r}3 \\
10\end{array}$ & $\begin{array}{l}2.9(0-6.5) \\
5.5(3.6-7.4)\end{array}$ & $\begin{array}{l}61.95 \\
52.96\end{array}$ & & $\begin{array}{l}1.2(0-4.8) \\
3.5(1.9-5)\end{array}$ & $\begin{array}{c}0 \\
58.81\end{array}$ & & $\begin{array}{l}1.1(0-2.5) \\
1.2(0.3-2)\end{array}$ & $\begin{array}{c}0 \\
47.09\end{array}$ & \\
\hline Study design & & & & 0.42 & & & 0.004 & & & 0.93 \\
\hline $\begin{array}{l}\text { - Case series } \\
\text { - Cohort } \\
\text { - Randomized } \\
\text { control }\end{array}$ & $\begin{array}{r}11 \\
1 \\
1\end{array}$ & $\begin{array}{l}4.2(2.4-6.1) \\
8.5(5.7-11.2) \\
5.7(0-12.2)\end{array}$ & $\begin{array}{c}60.3 \\
0 \\
0\end{array}$ & & $\begin{array}{l}2.6(1.6-3.7) \\
7.3(4.8-9.9) \\
3.8(0-9.1)\end{array}$ & $\begin{array}{c}19.27 \\
0 \\
0\end{array}$ & & $\begin{array}{l}1.3(0.1-2.1) \\
1.1(0.1-2.1) \\
1.9(0-5.6)\end{array}$ & $\begin{array}{c}42.83 \\
0 \\
0\end{array}$ & \\
\hline \multicolumn{4}{|l|}{ Setting } & 0.67 & & & 0.25 & & & 0.13 \\
\hline $\begin{array}{l}\text { - Multicenter } \\
\text { - Single center }\end{array}$ & $\begin{array}{l}6 \\
7\end{array}$ & $\begin{array}{l}4.5(1.7-7.3) \\
5.3(2.7-8)\end{array}$ & $\begin{array}{l}81.12 \\
43.85\end{array}$ & & $\begin{array}{l}4.1(2.1-6.1) \\
2.6(0.9-4.2)\end{array}$ & $\begin{array}{l}56.37 \\
36.97\end{array}$ & & $\begin{array}{l}0.7(0.1-1.3) \\
1.8(0.4-3.2)\end{array}$ & $\begin{array}{l}20.88 \\
33.41\end{array}$ & \\
\hline \multicolumn{4}{|l|}{ Location } & 0.72 & & & 0.75 & & & 0.43 \\
\hline $\begin{array}{l}\text { - USA } \\
\text { - Non-USA }\end{array}$ & $\begin{array}{l}4 \\
9\end{array}$ & $\begin{array}{l}4.5(1.7-7.3) \\
5.2(2.7-7.7)\end{array}$ & $\begin{array}{l}42.46 \\
76.18\end{array}$ & & $\begin{array}{l}3(1.3-4.8) \\
3.5(1.5-5.4)\end{array}$ & $\begin{array}{c}0 \\
67.59\end{array}$ & & $\begin{array}{l}1.6(0.2-3.1) \\
1(0.2-1.8)\end{array}$ & $\begin{array}{l}22.99 \\
38.07\end{array}$ & \\
\hline \multicolumn{4}{|l|}{ Quality } & 0.042 & & & 0.004 & & & 0.93 \\
\hline $\begin{array}{l}\text { - Low } \\
\text { - Moderate } \\
\text { - High }\end{array}$ & $\begin{array}{r}11 \\
1 \\
1\end{array}$ & $\begin{array}{l}4.2(2.4-6.1) \\
5.7(0-12.2) \\
8.5(5.7-11.2)\end{array}$ & $\begin{array}{c}60.28 \\
0 \\
0\end{array}$ & & $\begin{array}{l}2.6(1.6-3.7) \\
3.8(0-4.9) \\
7.3(4.8-9.9)\end{array}$ & $\begin{array}{c}19.27 \\
0 \\
0\end{array}$ & & $\begin{array}{l}1.3(0.4-2.1) \\
1.9(0-5.6) \\
1.1(0.1-2.1)\end{array}$ & $\begin{array}{c}42.83 \\
0 \\
0\end{array}$ & \\
\hline \multicolumn{4}{|c|}{ Non-dysplastic BE included } & 0.26 & & & 0.31 & & & 0.07 \\
\hline $\begin{array}{l}\text { - No } \\
\text { " Yes }\end{array}$ & $\begin{array}{r}12 \\
1\end{array}$ & $\begin{array}{r}4.8(2.8-6.7) \\
11.1(0.2-22)\end{array}$ & $\begin{array}{l}70.64 \\
0\end{array}$ & & $\begin{array}{l}3.5(2-4.9) \\
1.4(0-5.2)\end{array}$ & $\begin{array}{c}54.21 \\
0\end{array}$ & & $\begin{array}{l}1.0(0.4-1.7) \\
11.1(0.2-22)\end{array}$ & $\begin{array}{c}30.82 \\
0\end{array}$ & \\
\hline \multicolumn{4}{|c|}{ Number of EGDs negative for IM and EN required to define CE } & 0.46 & & & 0.03 & & & 0.09 \\
\hline $\begin{array}{l}.1 \\
.2\end{array}$ & $\begin{array}{l}2 \\
1\end{array}$ & $\begin{array}{l}6.9(3.7-11.1) \\
8.5(5.7-11.2)\end{array}$ & $\begin{array}{l}0 \\
0\end{array}$ & & $\begin{array}{l}3.5(1.2-5.7) \\
7.3(4.8-9.9)\end{array}$ & $\begin{array}{l}0 \\
0\end{array}$ & & $\begin{array}{l}3.2(1-5.4) \\
1.1(0.1-2.1)\end{array}$ & $\begin{array}{l}0 \\
0\end{array}$ & \\
\hline \multicolumn{4}{|l|}{ Cardia biopsied } & 0.68 & & & 0.37 & & & 0.66 \\
\hline $\begin{array}{l}\text { - No } \\
\text { - Yes }\end{array}$ & $\begin{array}{l}7 \\
5\end{array}$ & $\begin{array}{l}45(1.7-8.3) \\
4.3(2.9-5.7)\end{array}$ & $\begin{array}{c}81.47 \\
0\end{array}$ & & $\begin{array}{l}4.1(1.5-6.7) \\
2.8(1.5-4.1)\end{array}$ & $\begin{array}{l}66.21 \\
17.2\end{array}$ & & $\begin{array}{l}1.1(0.4-1.8) \\
1.4(0-2.97)\end{array}$ & $\begin{array}{c}0 \\
57.3\end{array}$ & \\
\hline \multicolumn{4}{|c|}{ Surveillance biopsy protocol, cm } & 0.76 & & & 0.75 & & & 0.98 \\
\hline $\begin{array}{l}. \\
.\end{array}$ & $\begin{array}{r}2 \\
34\end{array}$ & $\begin{array}{r}2.3(0-6.4) \\
34(0.4-5.6)\end{array}$ & $\begin{array}{l}70.66 \\
0\end{array}$ & & $\begin{array}{l}2(0-4.7) \\
2.6(0-4.7)\end{array}$ & $\begin{array}{l}0 \\
0\end{array}$ & & $\begin{array}{l}1.3(0-3) \\
1.3(0-3.4)\end{array}$ & $\begin{array}{c}36.26 \\
0\end{array}$ & \\
\hline \multicolumn{4}{|l|}{ BE length $>3 \mathrm{~cm}$} & 0.51 & & & 0.46 & & & 0.87 \\
\hline $\begin{array}{l}\text { BE length }>3 \mathrm{~cm} \\
\text { - No } \\
\text { - Yes }\end{array}$ & $\begin{array}{l}4 \\
8\end{array}$ & $\begin{array}{l}3.9(2.4-5.3) \\
5(2-7.9)\end{array}$ & $\begin{array}{c}0 \\
69.98\end{array}$ & & $\begin{array}{l}2.3(0.9-3.8) \\
3.1(1.5-4.7)\end{array}$ & $\begin{array}{l}26.55 \\
10.57\end{array}$ & & $\begin{array}{l}1.2(0-2.8) \\
1.4(0.3-2.5)\end{array}$ & $\begin{array}{l}38.7 \\
26.09\end{array}$ & \\
\hline
\end{tabular}

EGD = esophagogastroduodenoscopy, IM = intestinal metaplasia, $E N=$ early neoplasia, $C E=$ complete eradication, BE= Barrett's esophagus, $P Y=$ patient-years, SRER= stepwise complete endoscopic resection.

Competing interests

Sachin Wani is supported by the University of Colorado Outstanding Early Scholars Program and AGA Takeda Research
Scholar Award in Gastroesophageal Reflux Disease and Barrett's esophagus. Other author disclosures - Nicholas Shaheen receives research funding from CSA Medical, Covidien, C2 Therapeutics, CDx Medical, and Interpace Diagnostics. 


\section{References}

[1] Pohl H, Welch HG. The role of overdiagnosis and reclassification in the marked increase of esophageal adenocarcinoma incidence. J Natl Cancer Inst 2005; 97: 142 - 146

[2] Lepage C, Rachet B, Jooste V et al. Continuing rapid increase in esophageal adenocarcinoma in England and Wales. Am J Gastroenterol 2008; 103: $2694-2699$

[3] Crane S], Locke GR3rd, Harmsen WS et al. Survival trends in patients with gastric and esophageal adenocarcinomas: a population-based study. Mayo Clin Proc 2008; 83: 1087-1094

[4] Polednak AP. Trends in survival for both histologic types of esophageal cancer in US surveillance, epidemiology and end results areas. Int J Cancer 2003; 105: 98 - 100

[5] Miros M, Kerlin P, Walker N. Only patients with dysplasia progress to adenocarcinoma in Barrett's oesophagus. Gut 1991; 32: 1441 - 1446

[6] Devesa SS, Blot W], Fraumeni JF et al. Changing patterns in the incidence of esophageal and gastric carcinoma in the United States. Cancer 1998; 83: 2049-2053

[7] Hur C, Miller M, Kong CY et al. Trends in esophageal adenocarcinoma incidence and mortality. Cancer 2013; 119: 1149-1158

[8] Shaheen NJ, Falk GW, lyer PG et al. ACG clinical guideline: Diagnosis and management of Barrett's esophagus. Am J Gastroenterol 2016; 111: 30 - 50, quiz 51

[9] Ronkainen J, Aro P, Storskrubb T et al. Prevalence of Barrett's esophagus in the general population: an endoscopic study. Gastroenterology 2005; 129: $1825-1831$

[10] Zagari RM, Fuccio L, Wallander MA et al. Gastro-oesophageal reflux symptoms, oesophagitis and Barrett's oesophagus in the general population: the Loiano-Monghidoro study. Gut 2008; 57: 1354-1359

[11] Wani S, Falk GW, Post J et al. Risk factors for progression of low-grade dysplasia in patients with Barrett's esophagus. Gastroenterology 2011; 141: 1179-1186, 1186 e1

[12] Rastogi A, Puli S, El-Serag HB et al. Incidence of esophageal adenocarcinoma in patients with Barrett's esophagus and high-grade dysplasia: a meta-analysis. Gastrointest Endosc 2008; 67: 394-398

[13] Wani S, Falk G, Hall M et al. Patients with nondysplastic Barrett's esophagus have low risks for developing dysplasia or esophageal adenocarcinoma. Clin Gastroenterol Hepatol 2011; 9: 220-227, quiz e26

[14] Hvid-Jensen F, Pedersen L, Drewes AM et al. Incidence of adenocarcinoma among patients with Barrett's esophagus. NEJM 2011; 365: $1375-1383$

[15] Bhat S, Coleman HG, Yousef F et al. Risk of malignant progression in Barrett's esophagus patients: results from a large population-based study. J Natl Cancer Inst 2011; 103: 1049-1057

[16] Wani S, Sharma P. Challenges with endoscopic therapy for Barrett's esophagus. Gastroenterol Clin North Am 2015; 44: 355 - 372

[17] Konda VJ, Waxman I. Endotherapy for Barrett's esophagus. Am J Gastroenterol 2012; 107: 827-833

[18] Wani S, Early D, Edmundowicz S et al. Management of high-grade dysplasia and intramucosal adenocarcinoma in Barrett's esophagus. Clin Gastroenterol Hepatol 2012; 10: 704-711

[19] Wani S, Drahos J, Cook MB et al. Comparison of endoscopic therapies and surgical resection in patients with early esophageal cancer: a population-based study. Gastrointest Endosc 2014; 79: 224-232e1

[20] Shaheen N], Sharma P, Overholt BF et al. Radiofrequency ablation in Barrett's esophagus with dysplasia. NEJM 2009; 360: 2277-2288

[21] Phoa KN, van Vilsteren FG, Weusten BL et al. Radiofrequency ablation vs endoscopic surveillance for patients with Barrett esophagus and low-grade dysplasia: a randomized clinical trial. JAMA 2014; 311: $1209-1217$
[22] Wolf WA, Pasricha S, Cotton C et al. Incidence of esophageal adenocarcinoma and causes of mortality after radiofrequency ablation of Barrett's esophagus. Gastroenterology 2015; 149: 1752 - 1761.e1

[23] Pech O, Behrens A, May A et al. Long-term results and risk factor analysis for recurrence after curative endoscopic therapy in 349 patients with high-grade intraepithelial neoplasia and mucosal adenocarcinoma in Barrett's oesophagus. Gut 2008; 57: 1200 - 1206

[24] Wani S, Komanduri S, Muthusamy VR. Endoscopic eradication therapy in Barrett's esophagus-related neoplasia: setting the bar right to optimize patient outcomes. Gastroenterology 2016; 150: $772-774$

[25] Gerke H, Siddiqui J, Nasr I et al. Efficacy and safety of EMR to completely remove Barrett's esophagus: experience in 41 patients. Gastrointest Endosc 2011; 74: $761-771$

[26] Chung A, Bourke M], Hourigan LF et al. Complete Barrett's excision by stepwise endoscopic resection in short-segment disease: long-term outcomes and predictors of stricture. Endoscopy 2011; 43: 1025 1032

[27] Anders M, Bahr C, El-Masry MA et al. Long-term recurrence of neoplasia and Barrett's epithelium after complete endoscopic resection. Gut 2014; 63: $1535-1543$

[28] Konda V], Gonzalez Haba Ruiz M, Koons A et al. Complete endoscopic mucosal resection is effective and durable treatment for Barrett'sassociated neoplasia. Clin Gastroenterol Hepatol 2014; 12: 2002 2010, e1-2

[29] Pouw RE, Seewald S, Gondrie J] et al. Stepwise radical endoscopic resection for eradication of Barrett's oesophagus with early neoplasia in a cohort of 169 patients. Gut 2010; 59: 1169-1177

[30] Shaheen NJ, Overholt BF, Sampliner RE et al. Durability of radiofrequency ablation in Barrett's esophagus with dysplasia. Gastroenterology 2011; $141: 460-468$

[31] Orman ES, Li N, Shaheen NJ. Efficacy and durability of radiofrequency ablation for Barrett's esophagus: systematic review and meta-analysis. Clin Gastroenterol Hepatol 2013; 11: 1245-1255

[32] Pasricha S, Bulsiewicz WJ, Hathorn KE et al. Durability and predictors of successful radiofrequency ablation for Barrett's esophagus. Clin Gastroenterol Hepatol 2014; 12: 1840 -1847e1

[33] Phoa KN, Pouw RE, van Vilsteren FG et al. Remission of Barrett's esophagus with early neoplasia 5 years after radiofrequency ablation with endoscopic resection: a Netherlands cohort study. Gastroenterology 2013; 145: 96 - 104

[34] van Vilsteren FG, Pouw RE, Seewald S et al. Stepwise radical endoscopic resection versus radiofrequency ablation for Barrett's oesophagus with high-grade dysplasia or early cancer: a multicentre randomised trial. Gut 2011; 60: 765 - 773

[35] Gupta M, Iyer PG, Lutzke L et al. Recurrence of esophageal intestinal metaplasia after endoscopic mucosal resection and radiofrequency ablation of Barrett's esophagus: results from a US Multicenter Consortium. Gastroenterology 2013; 145: 79-86e1

[36] Vaccaro BJ, Gonzalez S, Poneros JM et al. Detection of intestinal metaplasia after successful eradication of Barrett's esophagus with radiofrequency ablation. Dig Dis Sci 2011; 56: 1996-2000

[37] Moher D, Liberati A, Tetzlaff J et al. Preferred reporting items for systematic reviews and meta-analyses: the PRISMA statement. Ann Intern Med 2009; 151: 264-269, W64

[38] Downs SH, Black N. The feasibility of creating a checklist for the assessment of the methodological quality both of randomised and nonrandomised studies of health care interventions. J Epidemiol Community Health 1998; 52: 377-384

[39] DerSimonian R, Laird N. Meta-analysis in clinical trials. Control Clin Trials 1986; 7: $177-188$

[40] Agoston AT, Strauss AC, Dulai PS et al. Predictors of treatment failure after radiofrequency ablation for intramucosal adenocarcinoma in 
Barrett esophagus: A multi-institutional retrospective cohort study. Am J Surg Pathol 2016; 40: 554-562

[41] Le Page PA, Velu PP, Penman ID et al. Surgical and endoscopic management of high grade dysplasia and early oesophageal adenocarcinoma. Surgeon 2016; 14: 315-321

[42] Orman ES, Kim HP, Bulsiewicz WJ et al. Intestinal metaplasia recurs infrequently in patients successfully treated for Barrett's esophagus with radiofrequency ablation. Am J Gastroenterol 2013; 108: 187 195 , quiz 196

[43] Cotton CC, Wolf WA, Pasricha S et al. Recurrent intestinal metaplasia after radiofrequency ablation for Barrett's esophagus: endoscopic findings and anatomic location. Gastrointest Endosc 2015; 81: 1362 1369

[44] Small AJ, Sutherland SE, Hightower JS et al. Comparative risk of recurrence of dysplasia and carcinoma after endoluminal eradication therapy of high-grade dysplasia versus intramucosal carcinoma in Barrett's esophagus. Gastrointest Endosc 2015; 81: 1158 - 1166, e1-4

[45] Akiyama J, Roorda AK, Marcus SN et al. Erosive esophagitis is a major predictor for recurrence of Barrett's esophagus after successful radiofrequency ablation. Gastroenterology 2013; 144: S692

[46] Lada MJ, Watson T], Shakoor A et al. Eliminating a need for esophagectomy: endoscopic treatment of Barrett esophagus with early esophageal neoplasia. Semin Thorac Cardiovasc Surg 2014; 26: $274-284$

[47] Shue P, Kataria R, Pathikonda R et al. Factors associated with recurrence of Barrett's esophagus after completion of radiofrequency ablation. Gastroenterology 2013; 144: S697

[48] Fitzgerald RC, di Pietro M, Ragunath K et al. British Society of Gastroenterology guidelines on the diagnosis and management of Barrett's oesophagus. Gut 2014; 63: 7-42

[49] Spechler SJ, Sharma P, Souza RF et al. American Gastroenterological Association technical review on the management of Barrett's esophagus. Gastroenterology 2011; 140: e 18 - 52 , quiz e13

[50] Krishnamoorthi R, Singh S, Ragunathan K et al. Risk of recurrence of Barrett's esophagus after successful endoscopic therapy. Gastrointest Endosc 2016; 83: 1090 -1106e3

[51] Wani S, Komanduri S, Early DS et al. Determining effectiveness and recurrence post endoscopic eradication therapy (EET) in Barrett's esophagus: results from a multicenter cohort study. Gastroenterology 2016; 150: S178

[52] Fleischer DE, Overholt BF, Sharma VK et al. Endoscopic radiofrequency ablation for Barrett's esophagus: 5-year outcomes from a prospective multicenter trial. Endoscopy 2010; 42: 781-789

[53] Alvarez Herrero L, van Vilsteren FGI, Pouw RE et al. Endoscopic radiofrequency ablation combined with endoscopic resection for early neoplasia in Barrett's esophagus longer than $10 \mathrm{~cm}$. Gastrointest Endosc 2011; 73: $682-690$

[54] Caillol F, Bories E, Pesenti C et al. Radiofrequency ablation associated to mucosal resection in the oesophagus: experience in a single centre. Clin Res Hepatol Gastroenterol 2012; 36: 371 - 377

[55] Gupta N, Wani S, Hollander TG et al. Recurrence of disease after endoscopic eradication therapy (Eet) for Barrett's esophagus (BE) with high grade dysplasia (HGD) and early cancer (EC). Gastroenterology 2012; 142: S750 - S751

[56] van Vilsteren FG, Alvarez Herrero L, Pow RE et al. Radiofrequency ablation and endoscopic resection in a single session for Barrett's esophagus containing early neoplasia: a feasibility study. Endoscopy 2012; 44: 1096 - 1104
[57] Dulai PS, Pohl H, Levenick JM et al. Radiofrequency ablation for longand ultralong-segment Barrett's esophagus: a comparative long-term follow-up study. Gastrointest Endosc 2013; 77: 534- 541

[58] Ertan A, Zaheer I, Correa AM et al. Photodynamic therapy vs radiofrequency ablation for Barrett's dysplasia: Efficacy, safety and costcomparison. World J Gastroenterol 2013; 19: 7106-7113

[59] Haidry RJ, Dunn JM, Butt MA et al. Radiofrequency ablation and endoscopic mucosal resection for dysplastic Barrett's esophagus and early esophageal adenocarcinoma: outcomes of the UK National Halo RFA Registry. Gastroenterology 2013; 145: 87 - 95

[60] Korst JR, Santana-Joseph S, Rutledge JR et al. Patterns of recurrent and persistent intestinal metaplasia after successful radiofrequency ablation of Barrett's esophagus. J Thorac Cardiovasc Surg 2013; 145: $1529-1534$

[61] Strauss AC, Agoston T, Dulai PS et al. Radiofrequency ablation for Barrett's-associated intramucosal carcinoma: a multi-center followup study. Surg Endosc 2014; 28: 3366 - 3372

[62] Lada M], Watson T], Shakoor A et al. Eliminating a need for esophagectomy: endoscopic treatment of Barrett esophagus with early esophageal neoplasia. Semin Thorac Cardiovasc Surg 2014; 26: 274-284

[63] Le Page PA, Velu PP, Penman ID et al. Surgical and endoscopic management of high grade dysplasia and early oesophageal adenocarcinoma. Surgeon 2016; 14: 315-321

[64] Phoa KN, Pouw RE, Bisschops R et al. Multimodality endoscopic eradication for neoplastic Barrett oesophagus: results of an European multicentre study (EURO-II). Gut 2015; 65: 555-562

[65] Giovannini M, Bories E, Pesenti $C$ et al. Circumferential endoscopic mucosal resection in Barrett's esophagus with high-grade intraepithelial neoplasia or mucosal cancer. Preliminary results in 21 patients. Endoscopy 2004; 36: $782-787$

[66] Larghi A, Lightdale C], Ross AS et al. Long-term follow-up of complete Barrett's eradication endoscopic mucosal resection (CBE-EMR) for the treatment of high grade dysplasia and intramucosal carcinoma. Endoscopy 2007; 39: 1086-1091

[67] Lopes CV, Hela M, Pesenti C et al. Circumferential endoscopic resection of Barrett's esophagus with high-grade dysplasia or early adenocarcinoma. Surg Endosc 2007; 21: 820-824

[68] Chennat J, Konda VJA, Ross AS et al. Complete Barrett's eradication endoscopic mucosal resection: an effective treatment modality for high-grade dysplasia and intramucosal carcinoma - an American single-center experience. Am J Gastroenterol 2009; 104: 2684-2692

[69] Brahmania M, Lam E, Telford J et al. Endoscopic mucosal resection: early experience in British Columbia. Can J Gastroenterol 2010; 24: $239-244$

[70] Moss A, Bourk M], Hourigan LF et al. Endoscopic resection for Barrett's high-grade dysplasia and early esophageal adenocarcinoma: an essential staging procedure with long-term therapeutic benefit. Am J Gastroenterol 2010; 105: 1276-1283

[71] Conio M, Fisher D, Blanchi S et al. One-step circumferential endoscopic mucosal cap resection of Barrett's esophagus with early neoplasia. Clin Res Hepatol Gastroenterol 2014; 38: 81 - 91

[72] Belghazi K, van Vilsteren FG, Weusten BL et al. Long-term follow-up results of stepwise radical endoscopic resection for Barrett's esophagus with early neoplasia. Gastrointest Endosc 2016; 83: AB555

[73] Waxman I, Vennelaganti S, Vennalaganti P et al. Recurrence rates after multi-modality endoscopic eradication therapy for dysplastic Barrett's esophagus (BE) and early esophageal cancer: results from an international, multi-center consortium. Gastrointest Endosc 2016; 83: $\mathrm{AB} 121-\mathrm{AB} 122$ 
高兴

ำ ํํำ

急哭

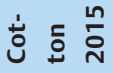

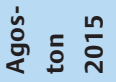

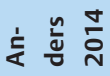

总哭

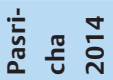

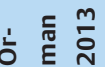

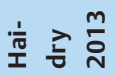

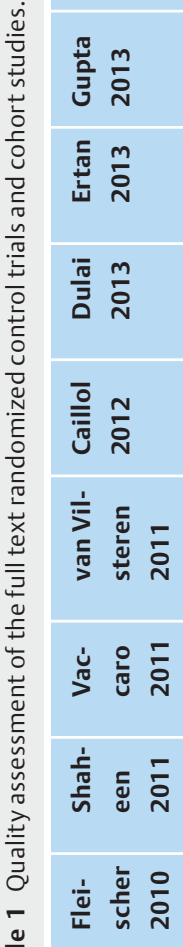

势高

䇏誉

高

흫

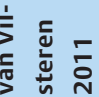

ํํำ

ฐ

咅高

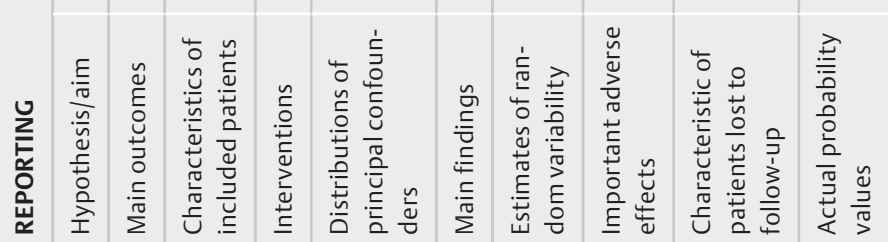
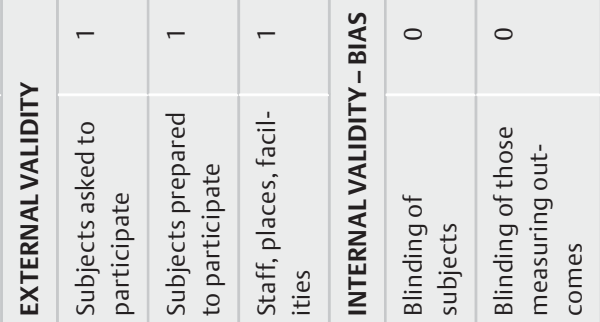


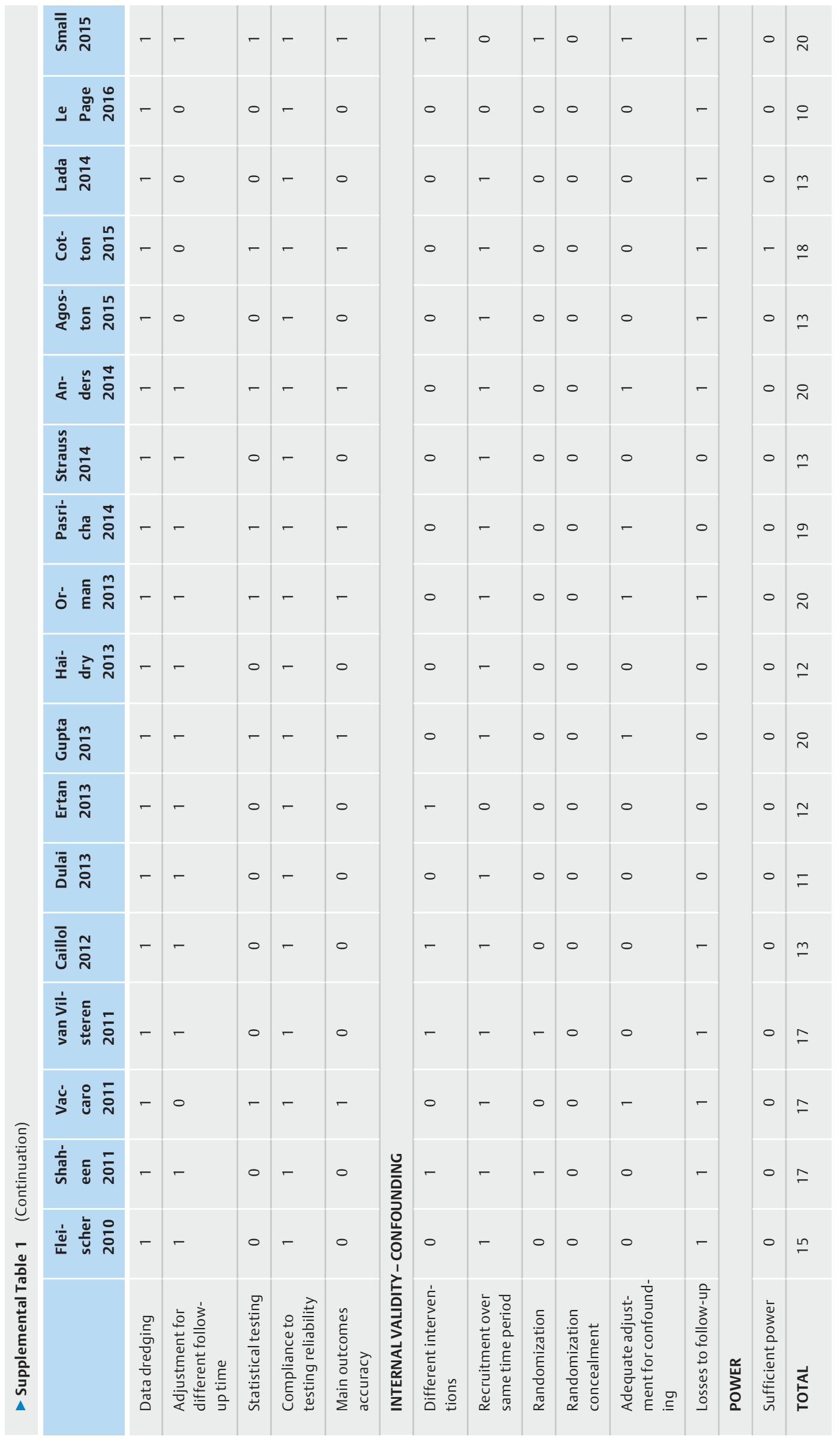

